\title{
LA CLASSE POLITICA DELLA TRANSIZIONE
}

\author{
di Luca Verzichelli
}

\section{4-1996. Un complesso periodo di rinnovamento}

La recente transizione italiana ha riaperto questioni che negli ultimi anni erano state trascurate dalle analisi politologiche, come le trasformazioni della classe politica e dei suoi modelli di selezione e carriera. Diversi contributi hanno evidenziato la nuova rilevanza assunta da tali questioni, confortati peraltro da un'ampia produzione pubblicistica che si è concentrata essenzialmente sulle caratteristiche socio-professionali dei nuovi politici e sul tasso di rinnovamento del personale parlamentare. Questo ritorno di interesse non chiarisce tuttavia quali obiettivi porre all'analisi, stretta tra una impostazione assolutamente de-

I dati utilizzati per questo saggio provengono dall'Archivio sulla Classe Politica Italiana, dell'Università degli Studi di Siena. L'aggiornamento dell'archivio, dopo le elezioni del 1996, è stato possibile grazie a un finanziamento del CNR (contratto 94.04271.CT09). La ricerca è inoltre inserita in un progetto di studio comparato sulle élite politiche finanziato dal programma Vigoni per gli scambi culturali tra Italia e Germania. Oltre ai consueti questionari, diretti al personale parlamentare, sono state consultate alcune fonti, relative ai dati biografici degli eletti e alle dirigenze partitiche, reperibili attraverso la rete Internet. In particolare, le banche dati della Camera e del Senato e $i$ siti ufficiali dei maggiori partiti politici italiani.

Il lavoro di attribuzione partitica degli eletti del 1996 è stato fatto di concerto con $i$ colleghi che banno contribuito a questa ricerca, seguendo criteri comuni di analisi. Nelle tabelle, tuttavia, bo utilizzato livelli diversi di disaggregazione. In particolare, bo distinto $i$ parlamentari del $\mathrm{Pds}$ (146 deputati e 88 senatori) sia rispetto al gruppo di partiti uniti nel cartello proporzionale della sinistra europea che rispetto al gruppo parlamentare della sinistra democratica. In questo modo lascio fuori i parlamentari laburisti, della rete, i cristiano sociali, $i$ comunisti unitari, $i$ socialdemocratici e $i$ laici di Ad che hanno aderito all'alleanza con la Quercia. Con lo stesso criterio bo separato i parlamentari del Ppi (54 e 29) dagli indipendenti, da quelli di Ud e da quelli del Pri che avevano aderito al cartello Popolari per Prodi. I parlamentari del $\mathrm{Ccd}$ e quelli del $\mathrm{Cdu}$ sono stati riaggregati, e $i$ loro dati sono comparati con quelli del solo Ced relativi al 1994. Nel caso di Rinnovamento e di Forza Italia bo invece mantenuto il livello di aggregazione più alto. Dunque le tre anime di Rinnovamento (Dini, Patto Segni e Socialisti Italiani) e le molte di Forza Italia sono tenute insieme nell'analisi che segue.

RIVISTA ITALIANA DI SCIENZA POLITICA / a. XXVI, n. 3, dicembre 1996 
scrittiva, che si limita a riportare le variazioni quantitative e qualitative della élite in un momento di ricambio evidente, ed un tentativo interpretativo che ancora oggi appare pieno di rischi, a causa della magmaticità in cui si muove il sistema politico italiano.

Esiste già una nuova classe politica e, se sì, quali caratteristiche ha? La difficoltà nel trovare risposte a tali interrogativi appare paradossale, in un paese dove era stata registrata per quasi mezzo secolo una tale continuità nei caratteri delle élites politiche da rendere frustrante il lavoro del ricercatore (Mastropaolo 1994). Eppure si tratta di un problema complesso, la cui soluzione deve essere cercata in fenomeni sociali e politici molto lontani rispetto alla data di inizio della transizione italiana, fenomeni che sono entrati infatti nel mirino degli studiosi. Facendo ordine tra i tanti suggerimenti, direi che il quadro teorico di riferimento per una riflessione sulla classe politica espressa durante la transizione italiana è delimitato da tre grandi argomenti di studio: in primo luogo, il nesso tra ricambio parlamentare e declino dei partiti di massa, un fenomeno già evidente alla fine dello scorso decennio, che ha spinto gli studiosi a mettere a punto nuovi possibili modelli teorici della forma partito (Katz e Mair 1995). Il secondo ed il terzo filone di studi da tenere in considerazione sono invece legati all'evoluzione del caso italiano, e si riferiscono rispettivamente alle interpretazioni della crisi recente e della transizione che ne è seguita, nonché agli effetti delle importanti riforme elettorali avvenute negli ultimi cinque anni.

Scendendo nel dettaglio, la crisi organizzativa dei partiti di massa è culminata, in Italia, con un crollo dei consensi che ha travolto i protagonisti del vecchio ordine politico e che, comunque, ha costretto i principali attori della opposizione a drastiche operazioni di ristrutturazione, sia a livello di classe dirigente che di programma (Morlino 1996). Dalla letteratura recente dedicata alla transizione italiana ci giungono invece numerosi spunti che utilizzano l'argomento del mutamento della classe politica con una certa varietà di toni e interpretazioni. Molte sono state le spiegazioni «negative», del cambiamento, basate cioè sulla sopraggiunta debolezza e inutilità del ceto politico preesistente (Pasquino 1995; Cotta e Verzichelli 1996). Altri autori sottolineano invece elementi di novità presenti già da qualche tempo, come per esempio l'idea della democrazia del leader, che avrebbe in qualche modo accelerato il declino della vecchia 
élite degli apparati partitici (Zincone 1995) o le crescenti richieste di una società in trasformazione già evidenziate dal voto di protesta o dall'astensionismo, nel quindicennio che precede le elezioni del 1992, e poi sfociate prepotentemente in un complesso anti-classe politica (Pizzorno 1996). La totalità dei contributi concorda però su una questione: il risultato del 1994, sotto il profilo del consolidamento dell'élite politica, era il punto di partenza della transizione e non quello di arrivo. La fine di un'epoca non aveva segnato con chiarezza i contorni del nuovo sistema, e la classe dirigente, definita frettolosamente dalla stampa come la protagonista della seconda repubblica, era attesa a nuove e decisive verifiche.

La rapida delegittimazione della classe politica del passato ha poi favorito, come sottolineato dalla totalità dei contributi sopra menzionati, un cambiamento incentrato in larga misura sulla reazione anti-partitocratica, imputando proprio alle vecchie formazioni politiche la responsabilità di aver trasformato la democrazia italiana da un sistema di partecipazione ad uno di protezione (Pizzorno 1996). Un primo strumento per osservare gli effetti della spinta anti-partitocratica sulla costruzione della nuova élite è contare semplicemente il numero di movimenti portati sul mercato elettorale, a partire dal 1992, che hanno rinunciato all'etichetta di «partito» e alla classica struttura organizzativa interna. Studi approfonditi hanno poi delineato come il sentimento anti-partitico abbia permeato l'intero spettro politico, obbligando molti attori a sottolineare una impostazione di rottura rispetto al passato e creando le condizioni per coalizioni nuove ed anomale (Bardi 1996). Più in generale è stato il «rinnovamento della politica» la parola d'ordine che ha accomunato le proposte per uscire dalla crisi e gli attori in competizione: quelli nuovissimi, quelli semi-nuovi e perfino alcuni navigatissimi leader del vecchio ordine scampati alla decimazione dei primi anni' 90.

Dall'altra parte dobbiamo tenere in conto i vincoli e le opportunità apparsi con le elezioni di questo ultimo quinquennio e non collegati direttamente con la crisi dei singoli partiti. In particolare, la nuova normativa elettorale (D'Alimonte e Chiaramonte 1994). A più riprese gli studiosi hanno sottolineato l'impatto di tali novità, che richiamo qui in modo sommario: la comparsa della esigenza maggioritaristica, con la prima, debole, riforma applicata nel 1992 (l'abolizione della preferenza multipla per l'elezione della Camera); il passaggio all'attuale sistema 
misto, applicato a partire dal 1994 , che ha incluso il ripristino, dopo ottant'anni, del collegio uninominale; le altre riforme in senso maggioritario, applicate alla legislazione elettorale degli enti locali, che hanno avuto effetti importanti nella re-distribuzione delle opportunità di carriera politica e nella organizzazione delle formazioni in lizza.

Quanto messo in luce dalla letteratura descrive dunque $\mathrm{i}$ presupposti di una vera transizione, capace di apportare profonde modificazioni, anche qualitative, all'intero processo di formazione e consolidamento della classe politica. Ricorrendo al modello a quattro variabili descritto da Pedersen nel suo classico lavoro sulle trasformazioni di lungo periodo delle élite danesi (1976), diremo che i diversi profili riscontrabili oggi nella composizione della classe politica possono essere dovuti ad «effetti mediati» sia in virtù dei mutamenti occorsi nelle strutture delle opportunità all'interno dei vari partiti, sia alla nuova distribuzione di seggi che consegue ad un forte trauma nel sistema partitico. Nel modello di Pedersen questi due fattori intervenienti, che potremo chiamare rispettivamente fattore intrapartitico e inter-partitico del mutamento nell'élite, variano insieme quando la quarta variabile, quella indipendente, è rappresentata da una complessa serie di cambiamenti sociali e politici. Questo può essere il caso della transizione italiana degli anni ' 90 , nella quale emerge tuttavia una particolarità: i mutamenti sociali e politici, pur profondi, non hanno avuto conseguenze rivoluzionarie: la costituzione del 1948 e la forma di governo parlamentare non hanno subito a tutt'oggi modificazioni, e anche le altre riforme annunciate, relative al decentramento amministrativo e fiscale, tardano a giungere in porto. L'incertezza della situazione genera una serie di retroazioni che incidono ulteriormente sui due fattori di mutamento della classe politica che menzionavo, tant'è che, nelle tre tornate elettorali ripetutesi in soli quattro anni, i cambiamenti sia a livello intra-partitico (cambiamenti di leadership, di organizzazione interna e di strategie) che a livello di sistema dei partiti (mobilità elettorale, fusioni, scissioni) hanno continuato a ripetersi invece di volgere verso la stabilizzazione.

Rispetto a ipotesi «forti» che prefigurano la scomparsa di quei modelli di reclutamento che hanno segnato l'esperienza storica della democrazia italiana, dovremo allora essere molto cauti; sarà utile invece differenziare i possibili scenari a seconda dell'impatto reale delle variabili sopra menzionate sul processo 
di stabilizzazione della nuova classe politica. In particolare, relativamente al ruolo del reclutamento partitico, si può ipotizzare l'accorciarsi del processo di socializzazione politica interna, con una incidenza minore delle carriere di partito rispetto all'acquisizione di posizioni pubbliche o professionali qualificanti. $\mathrm{Ma}$ sarà importante verificare se l'arretramento del ruolo partitico si arresta, con il procedere della transizione, e soprattutto se è presente in modo omogeneo nelle varie formazioni politiche.

Anche riguardo agli effetti della normativa elettorale maggioritaria si possono costruire ipotesi differenti: l'idea di un ribaltamento totale delle procedure tradizionali di selezione centralizzata delle candidature veniva già fortemente messa in dubbio dalle prime analisi dedicate alle elezioni del 1994, quando pure l'esigenza di rinnovamento e il disagio organizzativo dei partiti avrebbero potuto determinare il trionfo della territorializzazione (Mattina 1994; Lanza 1995). Tuttavia, il ricorso alla competizione maggioritaria ed alla candidatura uninominale ha prodotto alterazioni profonde nelle strategie partitiche (Katz 1996), e possiamo tornare a verificare ipotesi sugli effetti di un processo di apprendimento del sistema uninominale (Verzichelli 1994), che consisterebbero nell'emergere di una classe parlamentare relativamente slegata dal ceto politico nazionale, e maggiormente condizionata dalle strutture sociali ed economiche delle realtà locali.

Infine, dobbiamo misurare i reali effetti della complessa crisi italiana, e in particolare quelli del sentimento anti-partitocratico. Per le ragioni discusse sopra, la categoria dei parlamentari si è trasformata in pochi anni da simbolo della «stabilità italiana» al segmento di classe dirigente più suscettibile di cambiamento, per connotarsi (ancora oggi) come una classe politica della transizione, ossia un insieme ancora disomogeneo, all'interno del quale coesistono uomini politici vecchi e nuovi, elementi del reclutamento tradizionale ed evidenti tentativi di innovazione, ma anche personaggi «di passaggio», legati alla contingenza e non destinati ad entrare nella élite politica.

Gli studi successivi alle elezioni del 1994 avevano messo in rilievo soprattutto la dimensione del cambiamento, sollevando nel contempo forti dubbi circa la omogeneità e la stabilità della nuova classe politica, e generando, per forza di cose, più interrogativi che risposte. Dopo due tornate maggioritarie possiamo invece cominciare a cambiare prospettiva: il problema di oggi è quello di capire se la classe politica della transizione si stia con- 
solidando su basi differenti rispetto ai modelli di reclutamento e carriera predominanti fino a ieri, e se le sue varie facce presentino gradi diversi di innovazione. A tale fine la strada da percorrere è quella della doppia comparazione. $\mathrm{Da}$ una parte, tenendo sullo sfondo le caratteristiche note della classe politica preesistente, potremo descrivere quanto diversi rispetto al passato siano, a questo punto della transizione, i profili dei vari segmenti del ceto politico in via di formazione. Dall'altra parte un confronto di breve periodo tra i caratteri della classe parlamentare del 1996 con quella prodotta soltanto due anni or sono, nel momento culminante della crisi italiana, può dirci qualcosa di più chiaro circa le strategie perseguite e le potenzialità organizzative dei nuovi attori politici, nonché sui reali effetti di una legislazione maggioritaria-uninominale che, sia pure con possibili nuovi e importanti cambiamenti strutturali, è destinata a rimanere centrale nel nuovo ordinamento italiano.

Questo contributo si propone dunque di fare il punto sul processo di stabilizzazione della nuova classe politica e sulle sue caratteristiche socio-politiche, per cogliere le novità emerse rispetto ai modelli di reclutamento del passato e le variazioni significative presenti oggi al suo interno. L'analisi dei tassi di continuità della classe parlamentare degli ultimi anni sarà seguita dalla descrizione dei profili sociali e politici distinguibili nei suoi vari settori. Nella parte finale dell'articolo mi concentrerò invece sulle prospettive che si aprono alla classe politica dopo la transizione. In particolare vorrei chiarire se e come verrà sostituito quel professionismo politico che è stato l'elemento portante della classe parlamentare del passato (Cotta 1979; Mastropaolo 1993).

Alla ricerca della classe politica: ricambio parlamentare e fenomeni di ritorno

Veniamo dunque al primo punto, relativo alla stabilità della classe politica. I dati relativi alle elezioni del 1996 mostrano che, pur senza ripetersi il fenomeno del 1994, i livelli di ricambio parlamentare si mantengono elevati, evidenziando un turn over che oramai dall'inizio della crisi italiana (e da tre elezioni) si mantiene ben oltre gli standard del quarantennio precedente (fig. 1). Si può supporre che in occasione delle elezioni 1996 abbia giocato anche il fattore inter-partitico di rinnovamento, 


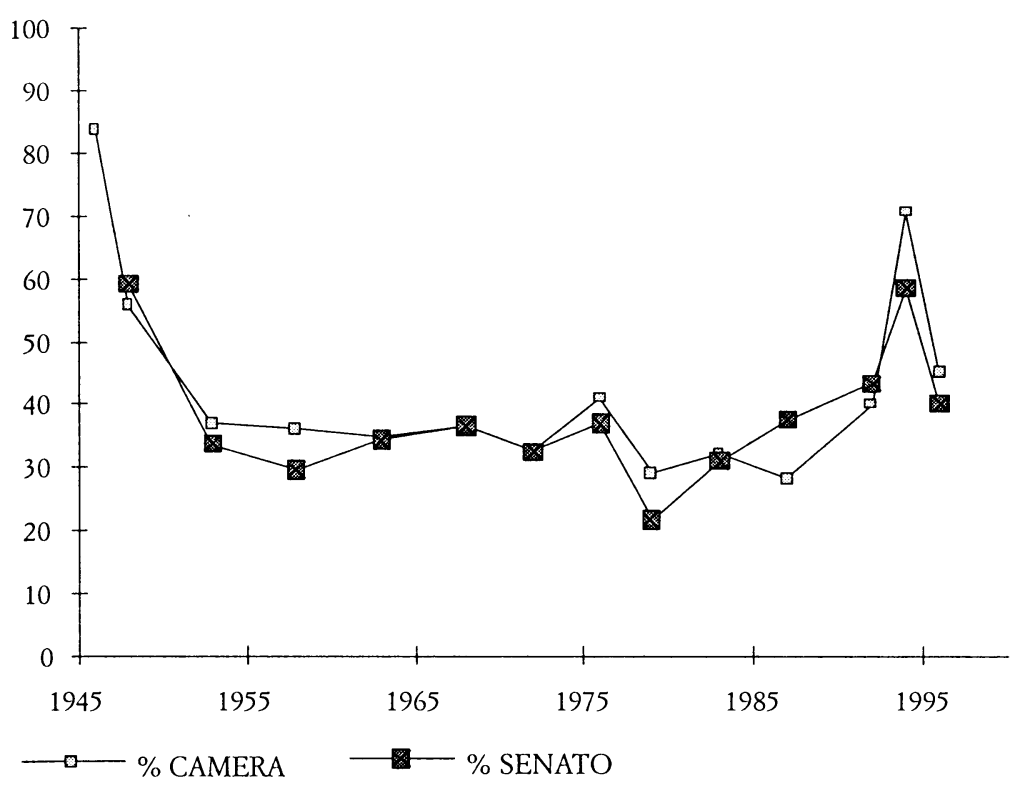

FIG. 1. Ricambio parlamentare 1946-1996.

cioè la bocciatura «sul campo» di una parte della classe politica dovuta allo spostamento di consensi dal polo di centro-destra, vincitore nel 1994, a quello di centro-sinistra. La classe politica italiana di oggi risponderebbe per così dire ad un fenomeno di assestamento del sistema partitico, attraverso il quale una parte del personale espresso dal cartello elettorale sconfitto è forzatamente costretta ad uscire di scena.

$\mathrm{Ma}$ a tale fattore bisogna aggiungerne altri di diversa natura, in particolare l'esigenza di un ulteriore avvicendamento all'interno della classe politica dovuto a scelte interne agli attori partitici, avvenute sin dal momento della definizione delle candidature. Per far luce su questo fenomeno ricostruiamo il destino dei parlamentari uscenti nel 1996 (tabb. 1 e 2): il cambiamento dei sistemi elettorali non consente, ovviamente, proficue comparazioni con il passato, ma è possibile ricercare in questi dati un orientamento tendenziale delle forze politiche oggi in campo circa il loro grado di controllo sui processi di selezione e di promozione delle candidature. In primo luogo si deve rilevare l'alta percentuale di uscita dal circuito della competizione elettorale, 
TAB. 1. Persistenza e abbandono della classe parlamentare Camera 1994-1996. (\% sui parlamentari della XII legislatura)

\begin{tabular}{lccccccccl}
\hline & Rc & Pds & Verdi & Ppi & Lega & Ccd & Fi & An & Tot. \\
\hline Rieletti 1 & 28 & 86 & 9 & 20 & 32 & 8 & 58 & 62 & 338 \\
& $(70,0)$ & $(69,9)$ & $(81,8)$ & $(58,8)$ & $(27,1)$ & $(38,1)$ & $(48,7)$ & $(58,5)$ & $(52,9)$ \\
Candidati & & & & & & & & & \\
$\quad$ non rieletti & 4 & 13 & - & 7 & 38 & 7 & 27 & 27 & 134 \\
& $(10,0)$ & $(10,6)$ & & $(20,7)$ & $(32,2)$ & $(33,3)$ & $(22,7)$ & $(25,0)$ & $(21,0)$ \\
Ritirati2 & 8 & 24 & 2 & 7 & 48 & 6 & 34 & 17 & 167 \\
& $(20,0)$ & $(19,5)$ & $(18,2)$ & $(20,7)$ & $(40,6)$ & $(28,6)$ & $(28,6)$ & $(16,0)$ & $(26,1)$ \\
\hline
\end{tabular}

1 Include i rieletti al Senato.

2 Include i deputati dimessi o deceduti durante la legislatura precedente.

TAB. 2. Persistenza e abbandono della classe parlamentare Senato1994-1996 (\% sui parlamentari della XII legislatura)

\begin{tabular}{lccccccccc}
\hline & Rc & Pds & Verdi & Ppi & Lega & Ccd & Fi & An & Tot. \\
\hline Rieletti & 8 & 52 & 6 & 19 & 18 & 3 & 16 & 28 & 162 \\
Candidati & $(42,1)$ & $(72,7)$ & $(46,2)$ & $(57,6)$ & $(30,0)$ & $(23,1)$ & $(44,4)$ & $(58,3)$ & $(50,3)$ \\
non rieletti & 3 & 1 & 1 & 2 & 11 & 6 & 5 & 3 & 33 \\
& $(15,8)$ & $(1,3)$ & $(7,7)$ & $(6,1)$ & $(18,3)$ & $(46,2)$ & $(13,9)$ & $(6,3)$ & $(10,2)$ \\
Ritirati & 8 & 21 & 6 & 12 & 31 & 4 & 15 & 17 & 127 \\
& $(42,1)$ & $(26,6)$ & $(46,2)$ & $(36,4)$ & $(51,7)$ & $(30,8)$ & $(41,7)$ & $(35,4)$ & $(39,4)$ \\
\hline
\end{tabular}

piuttosto accentuata in alcuni dei nuovi partiti di questo decennio (Lega Nord e Forza Italia). È facile mettere in relazione tale fenomeno con gli eventi e le trasformazioni interne ai partiti stessi, avvenuti nel concitato periodo che corrisponde alla XIII legislatura ${ }^{1}$, ma riscontri empirici più dettagliati evidenziano anche l'esistenza di precise strategie di rimodulazione dei criteri di

1 Gli assestamenti del sistema partitico prima dello scontro elettorale sono ricostruiti in dettaglio da Aldo Di Virgilio in questo stesso numero. Riguardo agli effetti dell'instabilità del sistema partitico sulla classe parlamentare durante la XII legislatura, rimando invece a Verzichelli (1996). La componente parlamentare che risente in modo maggiore di tale instabilità è ovviamente quella della Lega Nord, costretta a rinverdire le proprie liste dall'abbandono di molti parlamentari. Ma può essere interessante rovesciare il discorso, osservando quanto siano cambiati gli altri cartelli proprio per ospitare i transfughi dal partito di Bossi. Contiamo infatti 5 ex parlamentari leghisti oggi rieletti per il Polo, che ne aveva ricandidati altri cinque. A questi si aggiunge Pierluigi Pietrini che è stato rieletto per la lista Dini. Infine, altri sette ex deputati leghisti si sono presentati nel 1996 con partiti minori. 
selezione del personale, dovuti a cause diverse, come il cambiamento dei rapporti di forza interni nei rinnovati cartelli elettorali e l'alto grado di centralizzazione del processo di candidatura in alcune formazioni. Lo sforzo comune a tutti gli attori, in un quadro di grande incertezza e con un elevato numero di collegi «a rischio», è stato quello di preservare il futuro politico di una certa leadership, andando alla ricerca di una elezione sicura e sacrificando invece molti outsiders in collegi innaturali. Soltanto il $61 \%$ dei parlamentari «incombenti» si ricandida per mantenere il proprio collegio, e anche togliendo dal conto gli eletti del 1994 in quota proporzionale, oltre $1 / 4$ degli «incombenti maggioritari» non si presenta nel collegio vinto due anni prima ${ }^{2}$. A livello disaggregato colpisce il dato relativo a Forza Italia (ben 21 candidati incombenti spostati), tenendo anche conto che, per molti dei parlamentari azzurri, non si è trattato di un cambio di destinazione (dalla Camera al Senato) o di un semplice spostamento di collegio all'interno della stessa regione (tipica strategia adottata dalla Lega per favorire la rielezione di alcuni parlamentari rimastigli fedeli), ma di una operazione diretta dal centro che ha «paracadutato» molti leader visibili in regioni lontane da quelle originarie. Sotto questo profilo, certamente, l'adozione di un sistema uninominale non sembra aver ancora portato ad una territorializzazione della selezione politica (Fabbrini 1996), ed il vantaggio della incumbency, ammesso che di tale vantaggio si possa parlare, non è collegato tanto al potere ritagliato autonomamente dai parlamentari dentro il proprio collegio - come è stato dimostrato nel caso degli Stati Uniti ma anche in Canada, Germania e Giappone (Somit, Wildenmann, Noll, Römmele 1994) - ma, piuttosto, con le strategie adottate a livello centrale, dai partiti o dai «tavoli» coalizionali.

In generale le elezioni del 1996, nonostante la riduzione da tre a due grandi cartelli capaci di concorrere per i seggi uninominali, confermano un sistema partitico-parlamentare basato su circa dieci formazioni. Seguendo un discorso iniziato solo poco tempo fa, possiamo dire che la conferma di una parte cospicua del ceto politico affacciatosi sulla scena parlamentare nel $1992 \mathrm{e}$

2 Esattamente, gli incombenti che si presentavano in un collegio uninominale erano 606. Di questi 93 hanno corso in un collegio diverso rispetto a quello di due anni prima, 34 sono passati all'altro braccio del parlamento e 107 coloro che provenivano da una elezione in quota proporzionale. I «fedeli» al collegio sono dunque 372 . Ringrazio Alessandro Chiaramonte che ha condotto insieme a me questo rilevamento. 
nel $1994^{3}$ trasforma davvero gli sfidanti di ieri nella nuova élite, sebbene la seniority parlamentare rimanga molto meno marcata rispetto al passato (tab. 3). Differenze evidenti permangono tra i vari partiti, anche all'interno dei due schieramenti maggioritari. In particolare nel Polo per le libertà, dove una formazione come Forza Italia, ancora scarsamente istituzionalizzata dentro e fuori il parlamento, si contrappone a partiti connotati da un personale sicuramente più esperto. Da notare come sia i gruppi di Rifondazione comunista che del Pds mostrano una percentuale contenuta di senior members in entrambe le camere, ma ciò è da mettere in relazione con due diverse spiegazioni: nel caso di Rifondazione, partito nato da una componente oramai «sommersa» nel Pci e quindi scarsamente rappresentata in parlamento prima del $1992^{4}$, la selezione centralizzata, che fu tipica del modello comunista, funziona ancora: gli organi direttivi del partito stabiliscono esplicitamente anche il limite massimo di mandati per i propri rappresentanti. Nel dato relativo al Pds incide sicuramente una maggiore varietà del personale. La riduzione dei parlamentari esperti non è marcata come nel caso di Rc, ma l'abbassamento complessivo dell'anzianità di questo gruppo mostra il peso della componente giunta in parlamento dopo il 1992, ovvero della generazione di politici che ha trovato spazio nel partito dopo il congresso di Rimini.

Il consolidamento di una classe dirigente nuova non è tuttavia così facile. Il cambio della guardia è avvenuto, ma non ha ancora portato a quell'equilibrio tra fattori intra-partitici e inter-partitici necessario per una forte stabilizzazione. Il dato relativo al turnover nelle ultime tre tornate elettorali costituisce di per sé una evidente conferma di questa interpretazione.

Nel tentativo di produrre una lettura più dettagliata di questo difficile processo di consolidamento indicherei tre possibili direzioni: in primo luogo, facendo riferimento alle cause interpartitiche, il ricambio complessivo dell'élite politica degli ultimi anni può essere ricondotto agli effetti di una «tripla rivoluzione»: un primo momento, caratterizzato dallo sfaldamento parziale del vecchio sistema, con la comparsa di nuovi attori parti-

3 In totale tra gli attuali parlamentari troviamo 198 deputati che hanno esordito nel 1994 e 85 nel 1992. Al Senato questi due gruppi contano 95 e 57 unità. La percentuale di parlamentari entrati durante gli anni della crisi e oggi confermati in parlamento è dunque del 47,0 alla Camera e del 48,3 al Senato.

4 Ancor più evidente è questa tendenza dopo la scissione dei comunisti unitari, che ha tolto a Rifondazione comunista alcuni dei suoi più navigati parlamentari. 


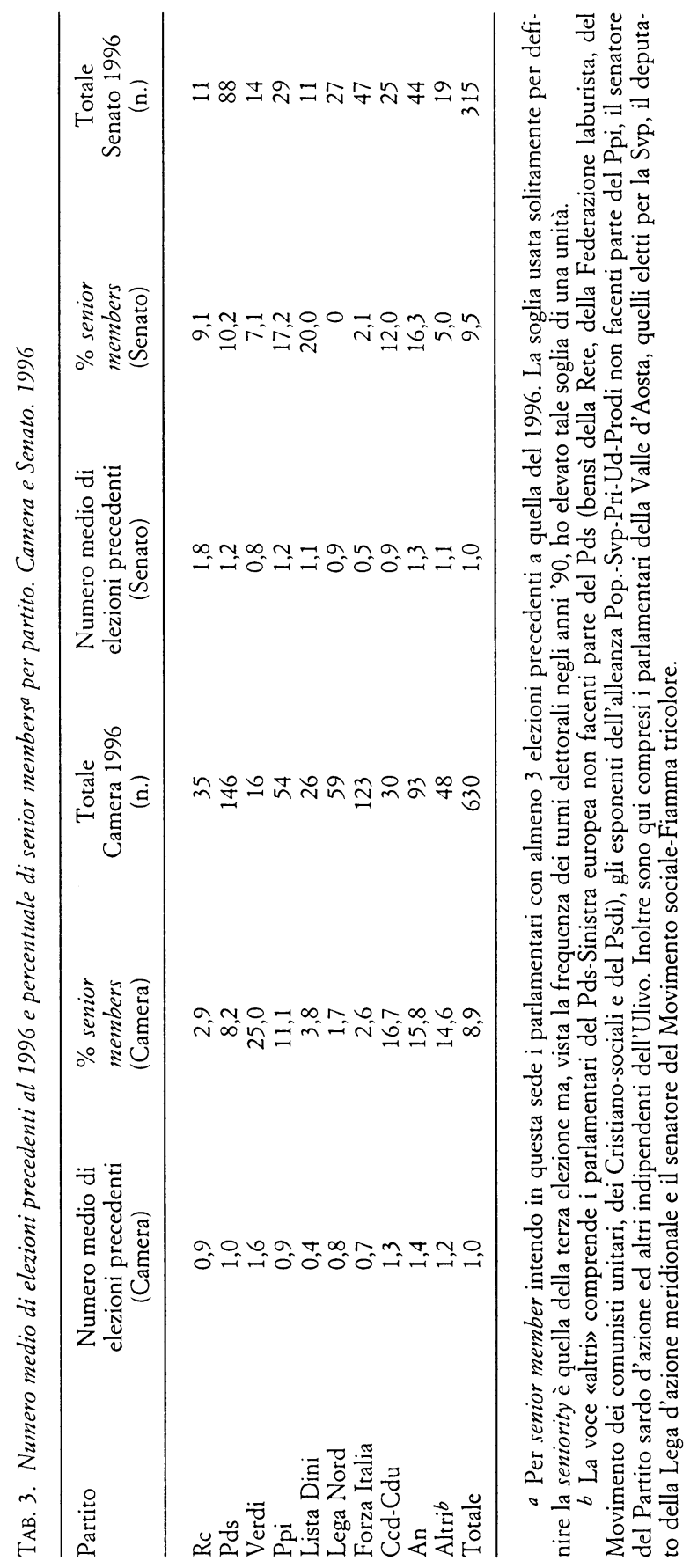


tici in alcune aree del paese (1992); una seconda fase, corrispondente alla vittoria del centro-destra nel 1994 e all'esordio di una formazione del tutto nuova (Forza Italia) che sfida, anche all'interno della propria area politica, il criterio tradizionale della selezione partitica; infine la rivincita del centro-sinistra nel 1996, con un ulteriore ritocco nella strutturazione dell'alleanza elettorale che implica non pochi cambiamenti anche a livello di classe politicas.

Un secondo elemento da tenere in considerazione è costituito dalla presenza di «fenomeni di ritorno» da parte di alcuni segmenti del vecchio ceto politico (figg. 2 e 3 ). Il dato generale circa i rieletti dopo un intervallo non è altissimo (40 parlamentari, pari al 4,2\% dell'universo), ma diventa significativo in alcune formazioni centrali (Ccd, Cdu e Ppi ed anche le componenti partitiche della Lista Dini), che utilizzano oggi il proprio potenziale coalizionale per riportare in parlamento esponenti, generalmente non di primo livello, già arrivati sulla scena politica nazionale prima del 1994. Anche altri partiti, da Rifondazione comunista a Forza Italia, non si sottraggono a questo fenomeno in parte spiegabile con il legame che unisce alcune formazioni, nuove o rinnovate, ad una leadership in qualche modo emarginata nei rispettivi partiti di origine ${ }^{6}$. Un'altra spiegazione può essere trovata nella debolezza organizzativa dei nuovi partiti che, soprattutto in quelle realtà locali dove settori del vecchio ceto politico mostrano ancora un buon rendimento elettorale, preferiscono reclutare i navigati uomini della prima repubblica. Emblematico è in questo senso il caso di De Mita, vincitore nel proprio collegio con una lista sostenuta dall'Ulivo. Ma sono molte le formazioni che rafforzano il proprio consenso con l'acquisizione di politici locali provenienti dai partiti storici.

5 La lettura più semplicistica di tale evoluzione ci porterebbe ad individuare un attore «nuovo» (la Lista Dini) da contrapporre ai partiti tradizionali. In realtà la situazione è più complicata. All'interno di Rinnovamento troviamo infatti formazioni e piccoli gruppi già sperimentati, che portano in parlamento alcune personalità dall'esperienza politica relativamente lunga. Il gruppo dei Popolari acquista un ristretto numero di parlamentari con una origine diversa da quella democristiana. Infine la compagine del $\mathrm{Pds}$, partito ancora in fase di ristrutturazione interna, affianca al personale politico proveniente dall'esperienza comunista, una serie di personalità della più vasta area dei progressisti già attivi in altre iniziative politiche degli ultimi anni.

6 Ciò è visibile soprattutto nei tre partiti di ispirazione cattolica e nella componente socialista della Lista Dini, dove osserviamo il ritorno di alcuni personaggi già in parlamento fin dagli anni ' 70 . Anche Fi recluta alcuni ex parlamentari del pentapartito, mentre Rc riporta in parlamento l'ex segretario di Democrazia Proletaria e tre ex parlamentari comunisti. 


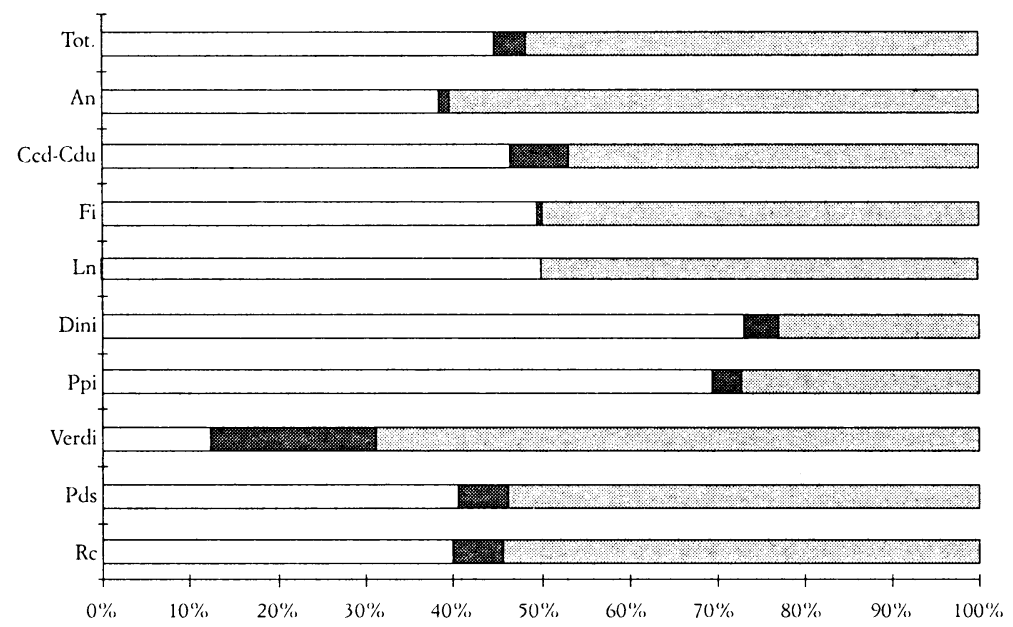
$\square$ neoeletti
rieletti dopo intervallo
${ }^{-}$rieletti

FIG. 2. Neoeletti e rieletti 1996. Camera dei deputati.

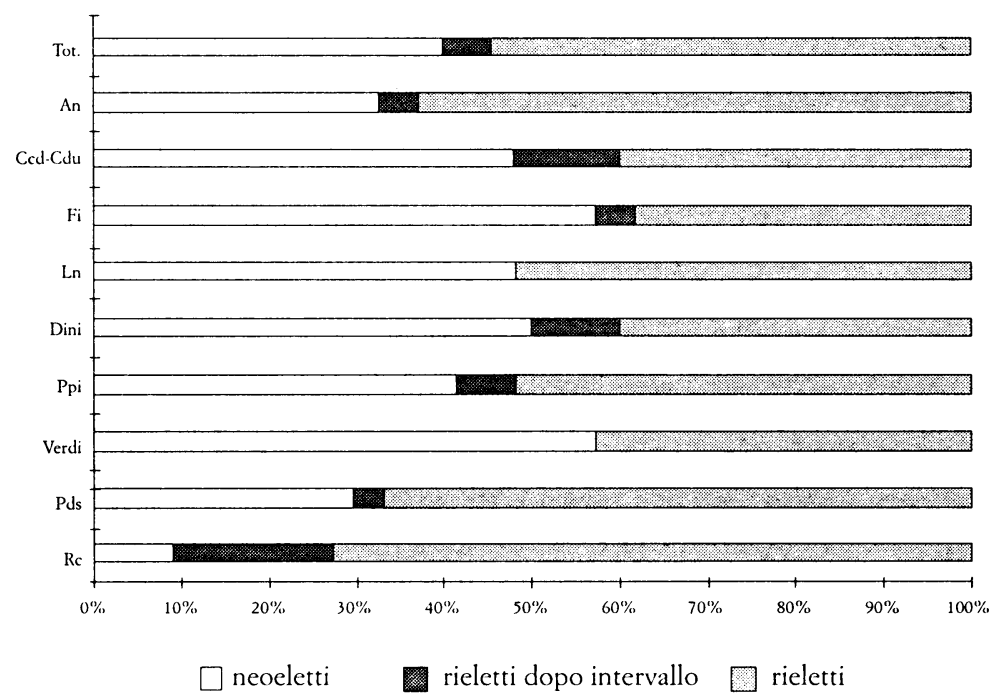

FIG. 3. Neoeletti e rieletti 1996. Senato della Repubblica. 
Infine, si deve sottolineare l'elevato abbandono, a distanza di soli due anni, da parte di una cospicua fetta della classe politica espressa nel momento della «rivoluzione» del 1994: circa il $26 \%$ dei deputati uscenti e addirittura il $40 \%$ dei senatori non si è ricandidato, e tale fenomeno è stato particolarmente accentuato nella Lega, nei partiti cattolici e in Forza Italia (tabb. 1 e 2). Ancora una volta si tratta di un dato che sta a metà tra il picco assolutamente straordinario del 1994 e la consolidatissima tendenza del periodo precedente, che si attestava alla Camera intorno al $20 \%$. Su questo dato influiscono tuttavia vari fenomeni, che agiscono in modo diverso sui vari gruppi: l'alto abbandono tra le fila del personale della Lega Nord, per esempio, è da mettere in relazione con le vicende interne al partito durante il biennio della XII legislatura, che hanno determinato la fuoriuscita di oltre un terzo del personale parlamentare in cari$\mathrm{ca}^{7}$. Gli altri scossoni subiti dal sistema partitico nel biennio tra le due tornate elettorali non avrebbero dovuto incidere sulle aspettative di rielezione dei parlamentari nei vari gruppi colpiti dalle scissioni ${ }^{8}$. Ciò nonostante il tasso di abbandono rimane, come si vede, piuttosto elevato. Particolarmente rilevante al riguardo il caso di Forza Italia, che «taglia» una larga parte dei parlamentari uscenti, spesso con un seguito di polemiche, nonostante l'atteso aumento della propria quota rappresentativa. In questo caso si conferma il controllo centralistico sulla selezione dei candidati (cfr. il saggio di Mattina e Tonarelli in questo numero). Percentuali elevate di abbandoni sono riscontrabili anche nel Ccd e in Rifondazione comunista. Al contrario, il Pds abbassa ulteriormente il tasso di selezione pre-elettorale sui parlamentari uscenti, allontanandosi, questa volta in modo più netto, dal modello tradizionale del Pci.

Nel complesso i dati sul ricambio e sull'esperienza del personale parlamentare mostrano che la nuova classe politica è giunta nella fase cruciale di un processo di istituzionalizzazione che può essere meno rapido di quello vissuto all'inizio dell'esperienza repubblicana, a causa della minore capacità di penetrazione degli attuali partiti politici e dei problemi indotti dalla riforma maggioritaria. Questo fatto rende ancora più im-

7 Esattamente si trattava di 42 deputati su 117 e 20 senatori su 60 (Verzichelli 1996).

8 In effetti gli ex popolari passati al Cdu hanno trovato un'ampia «quota» di candidature nelle liste del Polo e in quelle proporzionali comuni con il Ccd, mentre i Comunisti unitari sono entrati ufficialmente nella quota del Pds. 
portante il ricorso ad un'analisi dei suoi connotati socio-politici, come quella che segue nei prossimi paragrafi, ma apre anche nuovi interrogativi proprio sul problema dell'istituzionalizzazione. In particolare dovremmo tenere sotto controllo il grado di autonomia delle sub-strutture parlamentari e il ruolo della seniority nella selezione della leadership, sia politica che organizzativa, elementi che nel vecchio parlamento italiano avevano mostrato una grande continuità ${ }^{9}$. Per questi ultimi temi rinvio naturalmente ad ulteriori investigazioni.

\section{I protagonisti della transizione italiana: chi sono i nuovi politici}

L'analisi della classe politica può contare su un'ampia letteratura teorica e su una serie di ricerche empiriche che costituiscono importanti punti di riferimento. L'utilizzo di tali esempi può essere tuttavia fuorviante nella messa a punto di una ricerca dedicata ad una fase di trasformazione come quella di oggi: i mutamenti dell'ambiente sociale e politico possono infatti determinare, nel tempo, il ridimensionamento della capacità esplicativa di alcuni classici indicatori usati in questo approccio. E il caso di alcune variabili relative ai caratteri personali e sociali della élite, come il sesso, la scolarità, l'età media del personale eletto. La fisionomia della attuale classe politica, rispetto a quella di un tempo, appare molto più stabilizzata ed omogenea (per esempio la generale prevalenza di politici con una educazione universitaria) e comunque le variazioni riscontrate, nel tempo o nella distribuzione attraverso i vari gruppi partitici, sono dovute a fattori diversi rispetto al passato. In molti paesi occidentali l'analisi della struttura sociale degli eletti è tornata in auge negli ultimi due decenni, quando il declino del partito di massa e la trasformazione della cultura politica hanno determinato alcuni cambiamenti significativi. La classe parlamentare italiana, sotto questo profilo, ha recepito in ritardo ed in misura limitata la domanda di una maggiore rappresentanza femminile, mostrando invece un allargamento della scolarità universitaria in tutta l'élite politica, compreso il settore di essa proveniente dai vecchi partiti della sinistra di classe (Mastropaolo 1990; Mény 1995).

9 Si tratta dei classici indicatori dell'istituzionalizzazione parlamentare usati da Polsby e dagli altri studiosi che hanno trattato per primi questi concetti. Nei lavori di Cotta $(1976 ; 1979)$ troviamo una messa a punto specifica di tali indicatori e una serie di valutazioni su cosa essi mostrano relativamente al caso italiano. 
Diversi argomenti sono stati collegati alle peculiarità del caso italiano: per esempio il ricorso ad un reclutamento che coniugasse il personale partitico con elementi di visibilità sociale, se non la rinascita (per esempio nel Psi più recente) di elementi di selezione clientelare-localistica. Oppure lo scarso impatto della cultura post-materialista, che aveva invece inciso in modo più evidente nei sistemi partitici (e nella classe politica) di altri paesi. Ma quello che più ci interessa, nel fotografare complessivamente la situazione degli anni passati, è rilevare come i partiti storici, pur nelle loro differenze organizzative, abbiano determinato criteri di selezione del personale politico sempre più omogenei tra loro. L'evoluzione dei modelli di reclutamento parlamentare nel mezzo secolo della prima repubblica, racchiusi tra i tipi polari del modello d'apparato puro e quello partitico-clientelare (Cotta 1979) aveva dunque portato ad una classe politica dai tratti sociali non dissimili nei vari gruppi. Per questo motivo trascuro, in questa sede, l'analisi di lungo periodo delle caratteristiche pre-politiche del personale parlamentare, limitandomi a presentare, nelle tabelle 4 e 5 , i dati relativi alle ultime tre legislature: la colonna relativa al 1992 fotografa, per così dire, la situazione al termine del processo di evoluzione che ha visto protagonisti i partiti storici. Sono evidenti i segnali di invecchiamento della classe politica, soprattutto tra i partiti di governo ${ }^{10}$, ed emerge anche una prima differenza tra il personale del Pds, più giovane e scolarizzato, rispetto a quello di Rifondazione Comunista. Nella tornata elettorale successiva osserviamo significative differenze tra i vari partiti che, nonostante qualche assestamento, vengono confermate con le elezioni del 1996. Mi riferisco, in particolare, al dato relativo all'età media, che risente dello svecchiamento rispetto al decennio precedente (Lanza 1995), ma senza tornare ai livelli delle prime legislature repubblicane. Il fenomeno della stabilizzazione di una élite «nuova» ma non così giovane è del resto ancor più evidente quando osserviamo il dato relativo all'età di esordio in parlamento ${ }^{11}$. Viene confer-

10 Oltre al dato sulla Dc, riportato nelle tabelle, anche i partiti laici e socialisti presentavano all'inizio degli anni novanta un'età media molto elevata. Il Psi inoltre «avvicinava» la Dc anche nel tasso di scolarizzazione universitaria.

11 Il dato complessivo alla Camera si mantiene intorno ai 45 anni, in linea dunque con quello registrato nel 1992 e nel 1994, ma chiaramente superiore alle medie registrate in passato (Lanza 1995, 223). Concorrono, ad aumentare l'età media di prima elezione dei deputati, soprattutto Forza Italia (oltre 47 anni di media) ed il gruppo di Rinnovamento (49 anni). Tra i partiti legati in qualche modo a formazioni della prima repub- 
mato, inoltre, l'arresto del processo di allargamento della rappresentanza femminile, già evidenziato alla fine degli anni '80. L'eccezionale dato relativo alla Camera nel 1994 è dunque da imputare soltanto alla norma, che obbligava l'alternanza di uomini e donne nelle liste proporzionali. Sparita tale clausola, si torna ad una «normale» sotto-rappresentazione femminile, ripartita, come da tradizione, in modo piuttosto asimmetrico tra sinistra e destra. An, infatti, mostra in assoluto la più bassa percentuale di donne in parlamento ed anche i partiti cristiano-democratici di entrambi gli schieramenti si mantengono nettamente sotto la media ${ }^{12}$. Il Pds e Rifondazione sono invece i partiti con il gender gap parlamentare meno marcato. Relativamente all'istruzione, dobbiamo ancora una volta registrare come l'eredità dei partiti di classe si faccia sentire, ma con differenze meno marcate rispetto al passato: l'omogeneità di questi dati potrebbe essere imputata alla distribuzione di «vecchi» militanti partitici un po' in tutto lo spettro politico. Un caso evidente è quello della Lista Rinnovamento, nella quale i pochi parlamentari con un background politico (essenzialmente quelli espressi dai Socialisti Italiani di Boselli e Del Turco) bilanciano il gran numero di laureati candidati da Lamberto Dini e il suo entourage. Spariscono i micro-partiti parlamentari fatti quasi esclusivamente di intellettuali, che sfidavano le grandi formazioni di massa. $\mathrm{Ma}$, dall'altra parte, si raggiunge in tutte le liste un tasso di scolarizzazione molto elevato: con le sole eccezioni di Lega Nord e del partito neo-comunista, si assesta in ogni gruppo sopra il $60 \%$ con picchi di poco inferiori al $90 \%$ nei partiti moderati e cattolici.

Una tendenza da mettere in evidenza, rispetto a quanto genericamente descritto fino ad ora, è proprio quella che emerge nel personale della Lega Nord. Oramai da anni osserviamo come il partito di Bossi sia stato capace di formare una leadership diversa rispetto a quelle dei partiti tradizionali. In particolare, lo scarto nei dati sull'età media e sull'età di accesso in parlamento indica come questo settore della classe politica rappresenti, più che l'effetto di un salto generazionale, l'integrazione di parti della società sicuramente estranee alla politica prima

blica sono Ppi (47 anni) e An (oltre 45) a mostrare oggi l'età più elevata di accesso in parlamento.

12 Il dato relativo al Ppi nel 1994 (18,2\%) è infatti da imputare essenzialmente alla quota proporzionale. 


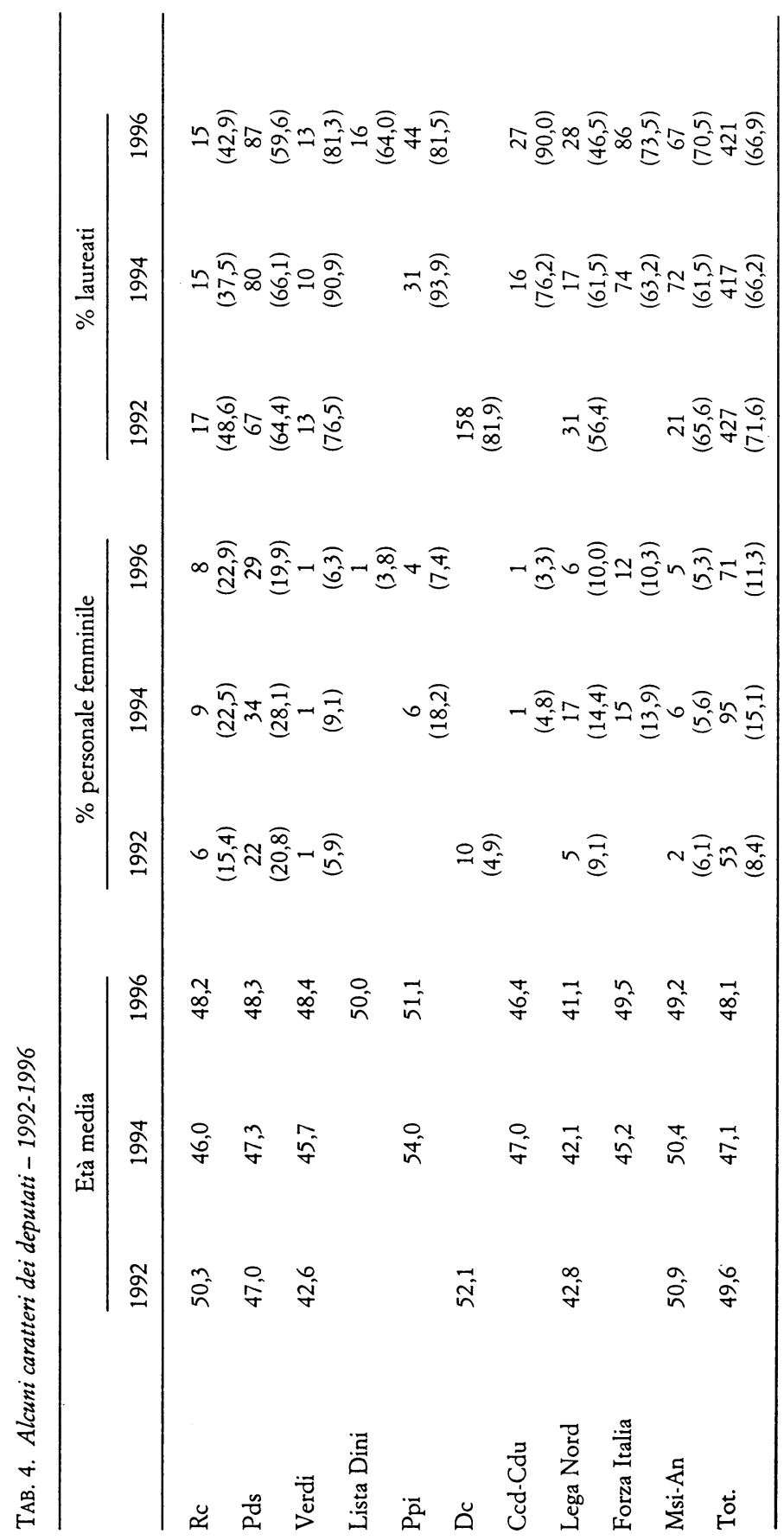




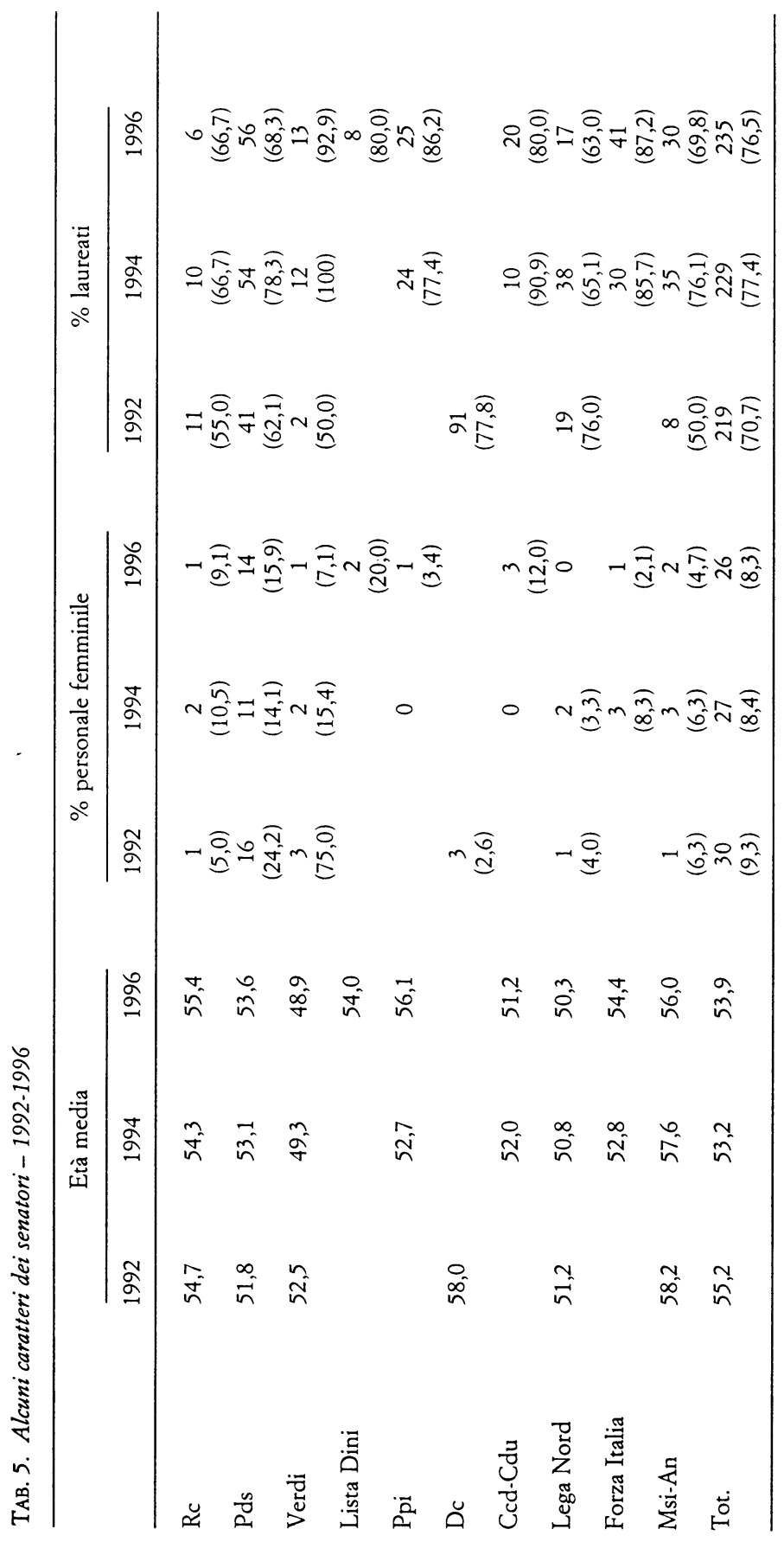


degli anni '90. L'altro elemento, quello del basso livello di scolarità universitaria, ci dice che, nella prepotente ascesa della leadership leghista, non sono gli elementi sociali e culturali a qualificare il candidato ma, evidentemente, la militanza all'interno dell'organizzazione. Su questo specifico punto tornerò tuttavia più avanti.

Quanto osservato sin qui evidenzia che, salvi gli effetti del «ricambio generazionale», avvenuto tra il 1992 e il 1994 (Lanza 1995), l'identikit sociologico della classe politica italiana non varia di molto rispetto al recente passato. Soltanto il personale leghista ha mostrato, fin dal suo esordio sulla scena politica, segnali rilevanti di novità, parzialmente confermate (soprattutto in merito all'età di entrata in parlamento), dal personale di Forza Italia. Altre forze, in particolare Rifondazione comunista, riflettono alcuni caratteri tipici dei loro referenti storici, mentre le formazioni nuove e rinnovate sia nel Polo che nell'Ulivo esprimono una classe parlamentare dai tratti sociologici stabili e non rivoluzionari rispetto ai partiti della prima repubblica. Si può dunque ipotizzare una qualche forma di persistenza dei classici meccanismi di selezione anche tra i settori più rinnovati della classe politica, quelli con basse percentuali di senior members (tab. 3) o di recuperati alla carriera parlamentare (figg. 2 e 3). In particolare nel caso di Rifondazione, partito dalla classe parlamentare «nuova» ma non giovane e dai caratteri sociali continuativi rispetto al passato; in misura inferiore il Pds e anche An, per il quale, d'altra parte, l'allargamento del consenso ha permesso la conferma di gran parte della classe politica preesistente e la promozione di molti quadri intermedi e outsiders del passato. In altri casi, soprattutto in Forza Italia, nei partiti cattolici e nelle liste di centro apparse nel 1994 e 1996, la continuità può essere dovuta ad una persistenza dell'élite, più che dell'apparato: gli outsiders del partito «antenato», gli esponenti di altri vecchi partiti recuperati dal nuovo movimento e i membri di élites non partitiche convertiti alla politica attiva potrebbero essere, l'analisi successiva ce lo dirà, i «nuovi» politici con caratteristiche sociali «antiche».

Un quadro completamente diverso si incontra osservando invece l'origine professionale dei parlamentari, che mostra evidenti segnali di variazione, anche rispetto al passato recente, ed una grande eterogeneità interna alla classe politica attuale (tabb. 6 e 7). Tale fenomeno ha recentemente interessato anche la stampa, che ha investigato su coloro che avrebbero dovuto 
sostituire i politici professionisti scomparsi dopo le tornate elettorali del 1992 e 1994. Alcuni elementi evidenziati dagli osservatori sono validi e comprovati dalla ricerca empirica: per esempio l'emergere e la persistenza, tra 1994 e 1996, di gruppi professionali «nuovi» per il parlamento, come i manager, i piccoli imprenditori, gli artigiani, i consulenti finanziari ed altri professionisti non forensi, o il rafforzamento dei professori universitari, dei medici e dei funzionari pubblici. Le conclusioni a cui si è pervenuti sono invece arbitrarie e spesso erronee. Il partito dei manager per esempio, identificato spesso come una delle anime del Polo per le libertà da contrapporre ad un partito di dipendenti pubblici o di professori universitari che vivrebbe all'interno dell'Ulivo, è in realtà composto da un gruppo certamente connotato sotto il profilo politico, ma che si identifica essenzialmente nel solo segmento di rappresentanti eletti dal partito di Berlusconi. La restante componente, tra i manager privati saliti sulla scena politica, è infatti distribuita tra tutte le altre formazioni. La variabilità nell'origine professionale dei parlamentari, all'interno dei blocchi maggioritari, è infatti evidente sia nel 1994 che oggi. Ma gli errori di interpretazione non sono dovuti solo alla lettura superficiale dei dati. Bisogna infatti tener presente la capacità esplicativa parziale di una variabile di background come l'origine professionale e la sua interazione con altri fattori. Per fare un esempio, l'importanza del carattere socio-occupazionale in un gruppo di accademici chiamati da un flash movement elettorale non può sicuramente essere valutata nello stesso modo rispetto a quella di intellettuali militanti che dividono la propria vita tra università e politica. In questo più ampio quadro interpretativo, i dati relativi all'origine professionale dei parlamentari italiani suggeriscono considerazioni diverse: le trasformazioni sociali degli ultimi decenni avevano già determinato, nelle ultime tornate elettorali dell'epoca proporzionale, alcuni piccoli, ma significativi, mutamenti del background socio-occupazionale ${ }^{13}$ dei politici. Il dato sulla crescita dei parlamentari provenienti dal settore pubblico è un buon indicatore di ciò, perché segna una netta inversione di tendenza rispetto

13 Rispetto ad una percentuale di funzionari ed impiegati pubblici (non contando dunque gli accademici) che tradizionalmente non superava il $15 \%$, dalla fine degli anni '70 abbiamo riscontrato un aumento di questa categoria, che già nelle due ultime legislature proporzionali aveva raddoppiato la propria rappresentanza, concentrandosi soprattutto nella Dc e nei partiti del centro-sinistra. 
ad una peculiare caratteristica della élite politica italiana, quella appunto del peso limitato del funzionariato pubblico, rilevata dalle analisi comparate (Mény 1995, 275). Quando, a tale trasformazione incrementale, si è aggiunta la crisi dei partiti storici, il ricorso a figure esterne è diventato una necessità alla quale quasi tutti gli attori in lizza hanno dovuto rispondere, e l'arrivo di una legge elettorale incentrata sul sistema uninominale ha infine giocato come fattore di accelerazione di questo processo. Tuttavia non dobbiamo dimenticare che la selezione della nuova classe politica rappresenta il punto di arrivo di un processo nel quale giocano (ancora) le organizzazioni che si fanno carico della campagna elettorale, e quindi in primo luogo i partiti (o i movimenti «non partitici» della transizione) in grado di riscuotere il consenso, e oggi, in seconda battuta, le «alleanze» elettorali. Quanto sta cambiando nella classe parlamentare ci può invece dire qualcosa circa la minore influenza di quel particolare ceto di professionisti della politica che rappresentava la vera peculiarità del caso italiano (Cotta 1979; Mastropaolo 1990; 1993). Ecco che il calo della percentuale di funzionari politicosindacali ${ }^{14}$ a tempo pieno si spiega come un effetto naturale del «dimagrimento» organizzativo dei partiti e, più in generale, la maggiore variabilità riscontrabile a partire dal 1994 nella origine professionale dei parlamentari indica il tentativo di rivolgersi a settori della società un tempo estranei alla politica attiva.

All'interno di questo quadro generale possiamo osservare alcune tendenze relative a specifici partiti, già delineate nei risultati del 1994 ed oggi sostanzialmente confermate. In particolare mi riferisco ai quattro partiti maggiori del panorama attuale, che incarnano nel proprio personale rappresentativo gradi diversi di innovazione. Da sinistra a destra troviamo: il partito della Rifondazione comunista, piuttosto continuativo con il passato relativamente al reclutamento parlamentare di funzionari politici e sindacali (tuttavia oramai contenuti sotto il $20 \%$ ) e di esponenti della classe lavoratrice; il Pds, che ancora traghetta in

14 Lo studio di Cotta (1979) riportava una percentuale di politici-sindacalisti di professione crescente fino al 1976, quando circa un terzo dei deputati presentava questa origine professionale. Il Pci dava il maggiore apporto in questo senso (con circa il 50\%) ma la crescita complessiva era da imputarsi anche all'aumento di politici professionisti nella rappresentanza democristiana e socialista. I nostri rilevamenti sugli anni ' 80 indicano che il tasso generale è sceso, attestandosi attorno al $20 \%$, mentre sono rimasti inalterati i rapporti tra i partiti: il Pci mostrava una più elevata incidenza di questa categoria, rispetto al Psi ed alla Dc. 


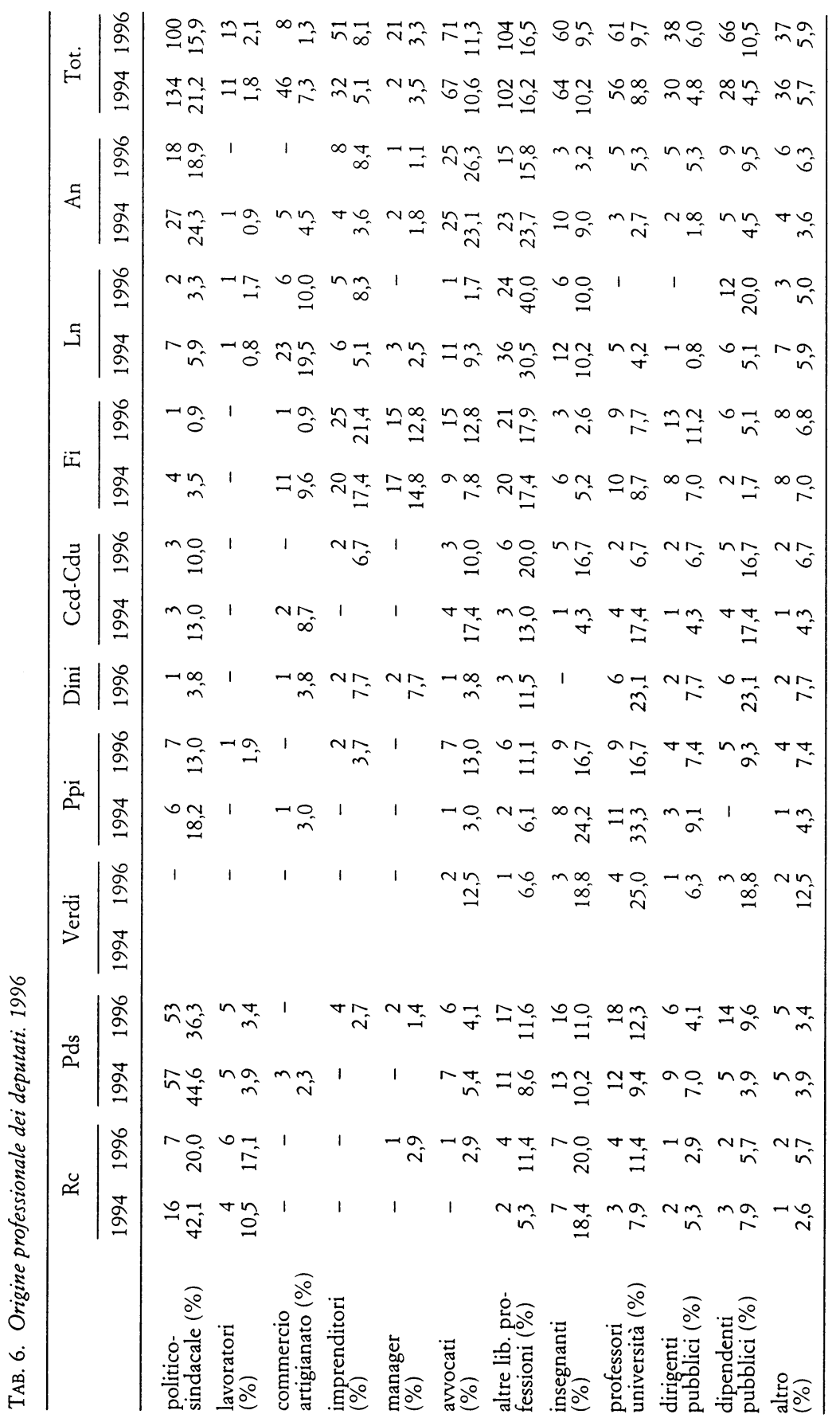




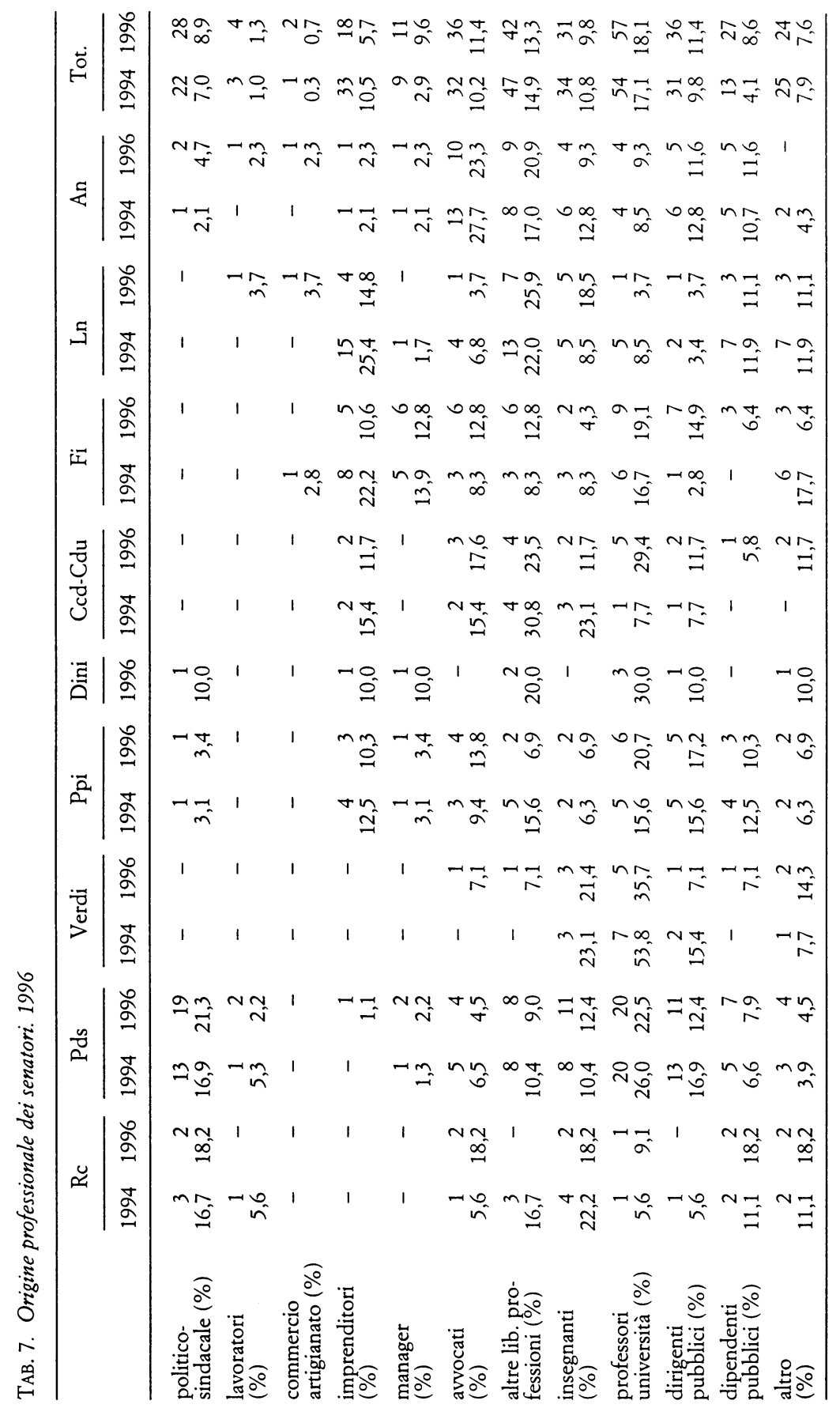


Parlamento una nutrita schiera di professionisti politici, inglobando tuttavia nella propria rappresentanza ${ }^{15}$ un personale dai tratti sociologici assai differenti rispetto al tradizionale apparato comunista: i professionisti, gli accademici e i dirigenti pubblici costituiscono nel complesso oltre un terzo dei deputati e circa metà dei senatori del maggiore partito della sinistra; Forza Italia, che conferma una classe parlamentare costruita essenzialmente sul ceto industriale e manageriale, continuando a reclutare personaggi professionalmente legati al gruppo di Berlusconi ${ }^{16}$; infine An, il cui personale rappresentativo sembra ancora legato alle categorie sociali tradizionalmente «visibili», in particolare la figura del professionista legale. Rispetto a questo quadro, ancora una volta dobbiamo rilevare l'eccezionalità del personale eletto dalla Lega Nord che si distingue sia rispetto ai partiti storici che a quelli nati dopo la sua comparsa. Inoltre, altra caratteristica della classe parlamentare leghista è quella di contrapporsi al complesso dei rappresentanti dell'Ulivo per l'evidente sovra-rappresentazione dei ceti produttivi del privato, ma anche ai due profili tipici riscontrati nel Polo, quello «manageriale» di Forza Italia e quello forense tipico in An. In questo caso si conferma infatti una classe politica vicina ai produttori della media e piccola borghesia: artigiani, commercianti, piccoli imprenditori, altri liberi professionisti, consulenti.

15 Giova ricordare ancora che i dati nelle tabelle sono calcolati solo sui parlamentari che abbiamo attribuito al Pds. A questi andrebbero aggiunti gli indipendenti di area e gli esponenti dei partiti vicini al Pds che hanno aderito alla lista proporzionale della sinistra europea e poi ai gruppi parlamentari della sinistra democratica (172 deputati e 98 senatori).

16 E difficile quantificare la portata di quello che potrebbe essere definito il «partito azienda» all'interno dei gruppi parlamentari di Fi. Utilizzando un criterio restrittivo (contando cioè soltanto gli uomini impiegati nel gruppo di Berlusconi) non più di quin. dici parlamentari erano presenti nel 1994 . Ma a questi andavano aggiunti personaggi in qualche modo legati alle sorti dell'impero economico del cavaliere (legali, consiglieri economici, giornalisti della Fininvest, ecc.). Oggi la situazione non sembra essere cambiata, e l'entrata in parlamento di esponenti di punta della Fininvest (Dell'Elce e Dell'Utri) o di altri collaboratori strettissimi di Berlusconi (Rivolta, Possa) mostra il tentativo di forzare ancora l'«aziendalizzazione» del personale da parte di Gianni Pilo, responsabile per la selezione del 1996. D'altra parte, ancora una volta la pressione da parte del core aziendale viene contrastata da una serie di forze interne al movimento, evidenziando le divisioni e le debolezze di un partito «elettorale», ma con evidenti connotati che richiamano altri modelli partitici (Gray e Howard 1995; Maraffi 1996). 


\section{La socializzazione politica dei parlamentari}

L'analisi della composizione professionale della nuova classe politica ridimensiona dunque le ipotesi che sostengono la sostituzione integrale del ceto di politici professionisti con un composito gruppo di amateurs giunti in ordine sparso direttamente dalla società civile e paracadutati in parlamento dalle riforme elettorali e dalla creatività degli italiani: le modifiche che abbiamo notato, rispetto al passato, risentono di impostazioni e strategie diverse da partito a partito, mescolando spesso elementi di novità con caratteri tradizionali di alcuni settori della classe politica. Inoltre non dobbiamo dimenticare le trasformazioni sociali degli ultimi anni, che hanno sicuramente modificato il «bacino di reclutamento» di ogni formazione. Tuttavia ci sono elementi sufficienti per pensare che il nuovo gruppo dirigente possa consolidarsi sulla base di requisiti piuttosto diversi rispetto al passato. Per verificare tale ipotesi è necessaria l'analisi di due variabili «classiche» di questo approccio, come quelle relative alle esperienze nella politica locale ed alle cariche partitiche ricoperte dagli eletti prima dell'esordio in parlamento. Le tabelle 8 e 9 rispondono a questa esigenza, sia pure relativamente al solo segmento di classe parlamentare espresso dalla Camera dei Deputati ${ }^{17}$. Tuttavia devo segnalare fin d'ora due limiti incontrati da questo approccio classico, ai quali dobbiamo porre rimedio con ulteriori analisi in profondità: in primo luogo, l'analisi della carriera partitica degli eletti veniva tradizionalmente condotta senza tenere conto di eventuali cambiamenti nelle affiliazioni politiche, fenomeno praticamente inesistente fino a qualche anno or sono. Oggi invece diventa importante osservare se la classe dirigente espressa dalle nuove formazioni proviene o meno da una socializzazione politica precedente e quanto lunga sia stata, eventualmente, tale esperienza. Il secondo ri-

17 I dati sin qui riportati, che presentano una comparazione tra Camera e Senato, confermano comunque ancora una volta la sostanziale omogeneità nei caratteri nei due settori. I pochi scostamenti fin adesso evidenziati sono generalmente riconducibili alle diverse condizioni elettorali (diversi limiti di età per l'elettorato passivo e minor numero di seggi in competizione). Restano da valutare, e la mancanza di dati approfonditi non consente di farlo in questa sede, le conseguenze della assenza, nel Senato, di una classe politica puramente proporzionale, ovvero eletta tramite liste di partito a livello circoscrizionale. Seguendo le ipotesi di questo saggio si dovrebbe pensare ad una minore presenza, nella camera alta, di candidature volute dal centro e di politici inadatti alla competizione maggioritaria. 
schio in cui incorriamo, utilizzando i classici indicatori della socializzazione politica, è quello di non cogliere il nuovo peso rivestito da alcune cariche elettive (e non) negli enti locali: l'introduzione di nuovi sistemi elettorali, basati sull'elezione diretta dei vertici esecutivi e ispirati al principio maggioritario ha infatti sicuramente modificato la struttura delle opportunità offerte tradizionalmente al politico in carriera, rivalutando soprattutto le posizioni ad elezione diretta rispetto a quelle ottenute per nomina (per esempio gli assessorati locali).

Rimanendo tuttavia sui dati generali, gli unici disponibili per il momento, le tendenze già emerse nel 1992 e poi, molto più chiaramente, due anni dopo, vengono confermate. L'esperienza negli organi nazionali di partito come pre-requisito per l'accesso parlamentare appare sicuramente meno rilevante rispetto alle serie storiche (tab. 8). Tale dato è particolarmente evidente se pensiamo che la frammentazione del parlamento attuale dovrebbe facilitare la «occupazione» delle Camere da parte dei gruppi dirigenti partitici nazionali, contando molte formazioni minori su un numero di seggi esiguo e spesso inferiore alla dimensione degli esecutivi nazionali. Eppure, oltre il 10\% in meno dei parlamentari proviene dalle top leadership partitiche. Andando ai dati disaggregati, notiamo come tutti i partiti abbiano abbassato il livello di proiezione delle leadership nazionali nel parlamento: la percentuale di deputati con un background partitico di vertice precedente alla prima elezione scende nettamente sotto il $50 \%$ nelle formazioni eredi dei partiti storici e si mantiene costantemente bassa in quelli nuovi. Le spiegazioni di tale fenomeno sono tuttavia diverse: in alcuni casi il processo di trasformazione intra-partitica ha snellito le leadership interne e rinnovato integralmente il suo personale ${ }^{18}$, mentre per quello che riguarda An è stato soprattutto l'aumento della rappresentanza parlamentare a determinare la diminuzio-

18 È questo il caso dei partiti post-democristiani ed in particolare del Ppi, la cui dirigenza nazionale è fatta per la gran parte di personaggi che avevano rivestito un ruolo minore nel vecchio organigramma. Anche una parte della dirigenza del Pds è stata promossa direttamente nell'élite nazionale nel momento della nascita del nuovo partito (per esempio Bassanini, Arlacchi, Salvi, Melandri). L'accesso in parlamento di molti di questi nuovi politici ha preceduto l'assunzione di cariche nazionali di partito, evidenziando molto la fase parlamentare della carriera politica rispetto al modello del Pci (Cotta 1979). Il ruolo del rendimento elettorale, nel contesto del sistema uninominale, può giocare in questo senso, tuttavia il consolidamento organizzativo dei nuovi partiti potrebbe nuovamente livellare questo fenomeno. 
TAB. 8. Deputati con precedenti esperienze partitiche (valori percentuali)

\begin{tabular}{|c|c|c|c|c|c|c|c|}
\hline & 1976 & 1979 & 1983 & 1987 & 1992 & 1994 & 1996 \\
\hline $\begin{array}{l}\text { Rc } \\
\text { nessuna carica } \\
\text { cariche basse } \\
\text { cariche medie } \\
\text { cariche alte }\end{array}$ & & & & & $\begin{array}{l}12,9 \\
12,9 \\
29,0 \\
45,2\end{array}$ & $\begin{array}{l}12,5 \\
10,0 \\
27,5 \\
50,0\end{array}$ & $\begin{array}{l}17,1 \\
28,6 \\
17,1 \\
37,1\end{array}$ \\
\hline $\begin{array}{l}\text { Pci-Pds } \\
\text { nessuna carica } \\
\text { cariche basse } \\
\text { cariche medie } \\
\text { cariche alte }\end{array}$ & $\begin{array}{l}14,1 \\
12,7 \\
44,4 \\
28,2\end{array}$ & $\begin{array}{l}19,6 \\
14,1 \\
38,6 \\
27,7\end{array}$ & $\begin{array}{r}20,0 \\
8,2 \\
46,5 \\
25,3\end{array}$ & $\begin{array}{r}14,7 \\
9,8 \\
47,2 \\
18,3\end{array}$ & $\begin{array}{r}21,7 \\
4,8 \\
36,1 \\
37,3\end{array}$ & $\begin{array}{r}31,1 \\
5,9 \\
35,3 \\
27,7\end{array}$ & $\begin{array}{l}24,7 \\
29,5 \\
17,8 \\
28,1\end{array}$ \\
\hline $\begin{array}{l}\text { Psi } \\
\text { nessuna carica } \\
\text { cariche basse } \\
\text { cariche medie } \\
\text { cariche alte }\end{array}$ & $\begin{array}{c}9,6 \\
- \\
34,6 \\
55,8\end{array}$ & $\begin{array}{r}19,3 \\
1,8 \\
17,5 \\
61,4\end{array}$ & $\begin{array}{r}12,2 \\
1,4 \\
20,3 \\
66,2\end{array}$ & $\begin{array}{r}9,5 \\
3,2 \\
21,1 \\
66,3\end{array}$ & $\begin{array}{r}9,6 \\
1,2 \\
25,3 \\
63,8\end{array}$ & & \\
\hline $\begin{array}{l}\text { Verdi } \\
\text { nessuna carica } \\
\text { cariche basse } \\
\text { cariche medie } \\
\text { cariche alte }\end{array}$ & & & & & $\begin{array}{c}66,7 \\
6,7 \\
- \\
26,7\end{array}$ & $\begin{array}{r}63,6 \\
9,1 \\
- \\
27,3\end{array}$ & $\begin{array}{l}56,3 \\
18,8 \\
12,5 \\
12,5\end{array}$ \\
\hline $\begin{array}{l}\text { Lista Dini } \\
\text { nessuna carica } \\
\text { cariche basse } \\
\text { cariche medie } \\
\text { cariche alte }\end{array}$ & & & & & & & $\begin{array}{r}57,7 \\
15,4 \\
7,7 \\
19,2\end{array}$ \\
\hline $\begin{array}{l}\text { Ppi } \\
\text { nessuna carica } \\
\text { cariche basse } \\
\text { cariche medie } \\
\text { cariche alte }\end{array}$ & & & & & & $\begin{array}{r}54,5 \\
3,0 \\
15,2 \\
27,3\end{array}$ & $\begin{array}{l}35,6 \\
20,3 \\
15,3 \\
28,8\end{array}$ \\
\hline $\begin{array}{l}\text { Dc } \\
\text { nessuna carica } \\
\text { cariche basse } \\
\text { cariche medie } \\
\text { cariche alte }\end{array}$ & $\begin{array}{r}21,7 \\
4,2 \\
41,7 \\
31,5\end{array}$ & $\begin{array}{r}22,4 \\
5,4 \\
40,2 \\
32,0\end{array}$ & $\begin{array}{r}23,2 \\
7,3 \\
38,2 \\
31,4\end{array}$ & $\begin{array}{r}29,0 \\
7,4 \\
29,4 \\
34,2\end{array}$ & $\begin{array}{r}23,9 \\
7,2 \\
36,7 \\
32,2\end{array}$ & & \\
\hline $\begin{array}{l}\text { Ccd-Cdu } \\
\text { nessuna carica } \\
\text { cariche basse } \\
\text { cariche medie } \\
\text { cariche alte }\end{array}$ & & & & & & $\begin{array}{l}33,3 \\
33,3 \\
14,3 \\
19,1\end{array}$ & $\begin{array}{l}30,0 \\
40,0 \\
13,3 \\
16,7\end{array}$ \\
\hline $\begin{array}{l}\text { Forza Italia } \\
\text { nessuna carica } \\
\text { cariche basse } \\
\text { cariche medie } \\
\text { cariche alte }\end{array}$ & & & & & & $\begin{array}{r}86,3 \\
6,0 \\
4,3 \\
3,4\end{array}$ & $\begin{array}{r}74,4 \\
15,7 \\
5,0 \\
5,0\end{array}$ \\
\hline $\begin{array}{l}\text { Lega Nord } \\
\text { nessuna carica } \\
\text { cariche basse }\end{array}$ & & & & & $\begin{array}{l}32,7 \\
11,5\end{array}$ & $\begin{array}{l}37,6 \\
13,7\end{array}$ & $\begin{array}{l}31,0 \\
36,2\end{array}$ \\
\hline
\end{tabular}


cariche medie

cariche alte

Msi-An

nessuna carica

cariche basse

cariche medie

cariche alte

Tot. Camera

nessuna carica

cariche basse

cariche medie

cariche alte

$29,2 \quad 28,2$

$26,5 \quad 20,5$

15,5

17,2

$11,1 \quad 15,4 \quad 14,3$

3,7

3,7

81,5

$$
-
$$

$3,8 \quad 8,6$

2,9

6,9

$-$

21,0

18,9

74,3

3,4

$-$

12,4

21,1

47,6

9,5
50,5

17,

6,8

22,5

22,0

24,5

92,6

44,0

36,7

36,5

7,0

5,6

5,6

20,4

9,1

24,2

32,7

32,0

27,7

5,6

20,4

12,1

$\begin{array}{lllllll}39,5 & 37,0 & 40,4 & 42,7 & 46,7 & 26,5 & 27,0\end{array}$

Nota: Le cariche basse sono tutte quelle al livello locale, salvo la segreteria provinciale. Le cariche medie includono le segreterie provinciali e le cariche regionali. Le cariche alte includono quella di segretario regionale e, naturalmente, tutte quelle a livello nazionale.

TAB. 9. Deputati con esperienze elettive locali (valori percentuali)

\begin{tabular}{|c|c|c|c|c|c|c|c|}
\hline Partito & 1976 & 1979 & 1983 & 1987 & 1992 & 1994 & 1996 \\
\hline $\mathrm{Rc}$ & & & & & 57,5 & 50,0 & 40,0 \\
\hline Pci-Pds & 67,7 & 61,0 & 55,0 & 58,4 & 53,3 & 46,2 & 61,6 \\
\hline Psi & 71,2 & 59,6 & 64,0 & 70,5 & 70,1 & & \\
\hline Verdi & & & & 42,9 & 52,9 & 45,5 & 50,0 \\
\hline Lista Dini & & & & & & & 38,5 \\
\hline Ppi & & & & & & 27,3 & 47,5 \\
\hline Dc & 60,7 & 60,2 & 62,4 & 61,9 & 70,1 & & \\
\hline Ccd-Cdu & & & & & & 56,9 & 48,3 \\
\hline $\mathrm{Fi}$ & & & & & & 15,7 & 19,7 \\
\hline Ln & & & & & 60,0 & 30,5 & 55,2 \\
\hline Msi-An & 62,5 & 67,7 & 71,4 & 75,0 & 84,8 & 77,5 & 67,4 \\
\hline Tot. & 63,5 & 57,3 & 56,6 & 58,9 & 65,1 & 41,3 & 48,6 \\
\hline
\end{tabular}

ne della quota di dirigenti nazionali tra i deputati. Inequivocabile invece il dato relativo a Lega Nord e Forza Italia: due gruppi parlamentari numerosi con una scarsa presenza di dirigenti politici di vertice. Corre l'obbligo di chiarire subito che in queste formazioni, specialmente in Forza Italia, le dimensioni ridotte delle strutture esecutive rendono comunque basso il dato qui costruito. Tuttavia sono pochi coloro che giungono in parlamento dopo aver fatto parte della ristretta cerchia di una cinquantina di nomi che costituisce il vertice dei due movimen$\mathrm{ti}^{19}$. La differenza tra azzurri e leghisti sta nel percorso inverso

19 Le differenze terminologiche e di struttura tra questi due partiti e quelli tradizionali impongono problemi nella definizione della «leadership nazionale». Per i fini di 
(dal parlamento al partito): mentre i componenti dell'esecutivo leghista sono in larga misura provenienti dalla prima ondata di parlamentari del carroccio, una larga fetta della dirigenza politica di Forza Italia rimane estranea alla carriera parlamentare ${ }^{20}$.

Se andiamo invece ad analizzare tutte le cariche partitiche antecedenti alla prima elezione, seguendo le altre voci della tabella 8 , il discorso cambia: con la sola eccezione di Forza Italia, tutti i partiti presenti portano ancora oggi almeno un $50 \%$ di uomini con una esperienza attiva nelle proprie strutture interne o comunque in quei partiti del passato considerabili come i progenitori delle formazioni di oggi. An in particolare torna, dopo la flessione del 1994, ad una elevatissima percentuale di parlamentari socializzati politicamente: probabilmente una nutrita schiera di politici di lunga esperienza ma chiusi, fino ad oggi, dalla vecchia guardia missina e da una quota limitata di seggi. Sono le seconde linee del Msi a costituire la spina dorsale del nuovo gruppo parlamentare di destra, con un tasso di continuità rispetto al referente storico più alto di quello riscontrabile tra i partiti post-democristiani, che pure tornano a una rappresentanza parlamentare fatta per $2 / 3$ da uomini con esperienze partitiche. Il modello di selezione alternativo rispetto a quello dell'accesso partitico certamente non scompare, ma si ridimensiona ovunque, tranne che nei partiti che contendono ai postdemocristiani l'area centrale dello spettro politico (Forza Italia e la componente non socialista di Rinnovamento). Vale ancora la pena di sottolineare il dato relativo al movimento di Berlusconi: considerando che l'esigua percentuale di deputati azzurri con esperienza partitica è imputabile anche a parlamentari provenienti da Pli, Dc e dal Partito Radicale, prende forma un personale caratterizzato da una socializzazione politica estremamente debole. Completamente opposta è, questa volta, la tendenza rilevata nel gruppo della Lega Nord, che evidenzia una

questa analisi ho considerato cariche alte in Forza Italia quelle di membro del Comitato Politico e di coordinatore regionale. Per la Lega Nord invece l'intera Presidenza e la Segreteria federale, nonché la carica di segretario nazionale (corrispondente al segretario regionale degli altri partiti).

20 Come è noto il Comitato Politico di Forza Italia contava, in origine, soltanto cinque membri, due dei quali (Berlusconi e Martino) furono eletti nel 1994. Dopo l'allargamento a 11 componenti (maggio 1994) erano 4 i parlamentari presenti nel comitato (i due menzionati e i capigruppo Della Valle e La Loggia). Oggi sono una decina i membri del Comitato a sedere in parlamento. Alcuni dei restanti membri sono stati tuttavia eletti, nel 1994, al Parlamento Europeo (per es. Caligaris, Ligabue, Tajani). 
schiacciante maggioranza di deputati provenienti dai quadri intermedi, cioè dal cuore «locale» del partito.

Considerazioni non dissimili alle precedenti ci vengono dai dati sull'esperienza locale degli eletti (tab. 9). Registriamo infatti che la diminuzione di questo tipo di esperienza, evidenziata nel 1994, si è arrestata, riportando il dato generale intorno al $50 \%$, ovvero a metà strada tra la tendenza registrata negli anni ' 80 e il dato relativo alla prima elezione maggioritaria. Artefici di questa risalita sono soprattutto i gruppi del Pds e del Ppi, che immettono in parlamento un personale sperimentato attraverso le elezioni locali ${ }^{21}$. Anche la Lega tuttavia risale alla percentuale del 1992, quando, come adesso, eleggeva «da sola» una sessantina di deputati (contro i 117 del 1994). Cambia invece rispetto al passato l'intensità dell'esperienza amministrativa pre-parlamentare. Unendo agli elementi emersi dalla tabella alcune considerazioni provenienti da approfondimenti qualitativi, potremmo rilevare tre percorsi tipici dei parlamentari con precedenti cariche locali: quello più «tradizionale» della carriera pre-parlamentare, attraverso una serie piuttosto lunga di impegni elettorali ed amministrativi, quella di un gruppo di politici la cui carriera, interrotta dalla crisi degli anni '90, è ripresa direttamente dal livello parlamentare, dopo una fase di assenza da ogni scenario, e infine quella dei nuovi politici che hanno sperimentato esperienze elettive locali soltanto nell'epoca del maggioritario, e quindi a ridosso dell'entrata in parlamento. Con una certa approssimazione direi che in $\mathrm{Rc}$, Pds e An si concentra il primo gruppo di parlamentari ${ }^{22}$, nei partiti ex democristiani riscontriamo molti casi appartenenti al secondo tipo, mentre Lega, Forza Italia e i «cespugli» di centro-sinistra promuovono molti parlamentari del terzo tipo.

Una valutazione finale sulla socializzazione politica dei nuovi parlamentari potrà essere fatta soltanto alla luce di ulteriori dati, ma quanto oggi disponibile può già fornirci un indicatore di massima che è costituito dal background politico completo,

21 Dei nuovi deputati attribuiti al Pds ben 41 (68,3\%) mostrano una precedente esperienza nelle amministrazioni locali. Più basso, ma significativo, il dato tra i popolari: $21(51,2 \%)$. Simile invece la percentuale di nuovi eletti che non ha rivestito cariche di partito: $19(31,7 \%)$ per il Pds e $12(29,3 \%)$ per il Ppi.

22 A questo riguardo farei una distinzione ulteriore, vista la scarsa percentuale di ex amministratori locali tra i parlamentari di An. Ma tale differenza si motiva ovviamente con il fatto che il vecchio Msi rimaneva un partito escluso dall'esecutivo anche a livello locale. 
TAB. 10. Deputati con background politico (cariche elettive locali + incarichi di partito) precedente alla elezione. 1992-1996 (valori percentuali)

\begin{tabular}{lcrl}
\hline Partito & 1992 & 1994 & 1996 \\
\hline Rc & 52,5 & 45,0 & 40,0 \\
Pds & 42,5 & 43,7 & 54,8 \\
Psi & 60,9 & & 25,0 \\
Verdi & 47,1 & 9,1 & 30,8 \\
Lista Dini & & 15,2 & 39,0 \\
Ppi & 56,8 & & \\
Dc & & 42,9 & 41,4 \\
Ccd-Cdu & & 7,7 & 13,0 \\
Fi & 38,2 & 22,2 & 44,1 \\
Ln & 69,7 & 69,8 & 62,1 \\
Msi-An & 51,4 & 32,9 & 41,1 \\
Tot. & & & \\
\hline
\end{tabular}

Nota: Si computano qui tutte le cariche amministrative locali, da consigliere comunale a membro dell'esecutivo regionale.

ovvero dalla percentuale di parlamentari che presentano sia un'esperienza partitica che una locale precedenti all'elezione (tab. 10). Una tendenza di fondo sembra già chiara: i politici «esperti» si rafforzano superando la crisi del 1994 ma attestandosi ben al di sotto del $50 \%$ che costituiva il livello riscontrato alla fine della prima repubblica. Il personale della sinistra garantisce il tasso più elevato di socializzazione politica, grazie al personale di Rifondazione ed al settore di parlamentari identificabile con il $\mathrm{Pds}^{23}$, seguito dai partiti cattolici (intorno al $45 \%$ ) e da un centro-destra molto composito. Emerge ancora il balzo della Lega Nord verso i livelli di quattro anni fa, ulteriore segnale del ritorno ad un personale (e ad un criterio di reclutamento) più centrato sulla militanza partitica.

Verso un nuovo professionismo politico? La classe parlamentare in movimento

Torniamo alle questioni centrali a cui questa prima lettura dei dati sulla classe politica della transizione vuole dare risposta: esistono i presupposti per parlare di rinnovamento qualita-

23 Segnalo ancora una volta che il dato in tabella si riferisce solo ai 146 deputati da noi attribuiti al Pds. Tuttavia riaggregando tutti i 172 deputati della sinistra democratica il valore rimane al $51,5 \%$. 
tivo del personale parlamentare? Se sì, tali presupposti suggeriscono lo sviluppo di una tipologia di reclutamento diversa rispetto a quella espressa fino agli inizi degli anni '90? I dati passati in rassegna mostrano alcune tendenze di fondo che devono essere interpretate con la doppia lettura indicata in precedenza: quella di lungo periodo, che compara l'attuale volto della classe politica con la configurazione del passato, e quella di breve periodo, che confronta due momenti ravvicinati, cioè il 1994, culmine della transizione e della fase di ricambio parlamentare, con il 1996. Gli elementi da sottolineare sono i seguenti: in primo luogo, la fase di eccezionale rinnovamento, che ha attraversato l'ultimo quinquennio, tende ad esaurirsi, pur permanendo tassi di ricambio superiori rispetto all'epoca del proporzionale. I fenomeni di «riciclaggio» di segmenti della vecchia classe politica, enfatizzati da alcuni osservatori a ridosso delle elezioni, si riducono a pochi casi, tuttavia significativi se letti unitamente alla reimmissione nel circuito elettorale di una cospicua fetta di nuovi parlamentari socializzati dai vecchi partiti e forti di esperienze politiche precedenti. In particolare, si stabilizzano i dati circa le esperienze partitiche e quelle nelle amministrazioni locali soprattutto tra le formazioni politiche più organizzate.

La configurazione dell'insieme dei circa mille personaggi che oggi costituisce la classe parlamentare si stabilizza confermando alcuni connotati già evidenti nell'élite politica durante la fase finale della prima repubblica (la sotto-rappresentazione femminile ed un'età media intorno ai 50 anni), confermando nel contempo alcune novità emerse nel 1994. In particolare, mi soffermerei sul calo della quota di parlamentari con origini professionali politico-sindacali, sulla conferma di alcuni gruppi provenienti dalla società civile e dai ceti produttivi (accademici «puri», manager, dirigenti pubblici e privati, giornalisti non di partito), nonché sulla riduzione della fase pre-parlamentare di socializzazione politica, mostrata dal minor numero di parlamentari con incarichi nazionali di partito e dallo stabilizzarsi di una socializzazione politica comunque inferiore rispetto alla prima repubblica (tab. 10). In questo quadro complessivo si innestano evidenti differenze tra $i$ vari partiti, anche queste in via di stabilizzazione a giudicare dal confronto tra 1994 e 1996. Soprattutto l'analisi del background professionale mostra, a questo proposito, segnali di continuità che fanno pensare al consolidamento di una élite frammentata anche all'interno degli stessi cartelli maggioritari (Verzichelli 1995). 
Quanto evidenziato mi porta a confermare l'inaffidabilità di ipotesi forti circa il completo smantellamento dei criteri preesistenti di selezione del personale politico. La sostituzione della classe parlamentare, unico effetto certo della transizione italiana (unitamente con la destrutturazione del vecchio sistema partitico) non cancella necessariamente i vecchi percorsi di accesso. Le elezioni del 1996 portano, sotto questo profilo, soltanto un assestamento rispetto a quanto osservato due anni prima e confermano la tendenza, da parte di tutti i partiti «eredi» della prima repubblica, a produrre una classe politica di marca partitica (Recchi 1996). Tuttavia le novità emerse (ed oggi confermate) sono sufficienti per verificare più ipotesi sul mutamento, basate su fattori che giocano in modo diverso nei vari schieramenti. Alla prima delle due domande sopra formulate mi sento allora di rispondere in senso positivo. La stessa persistenza di un canale alternativo al reclutamento partitico, come quello presentato da Forza Italia, e di un modello comunque non assimilabile a quelli tradizionali (Lega Nord), spinge la risposta in questa direzione. Le esigenze (negative) di sostituzione del tradizionale ceto politico e quelle (positive) di ridefinizione di un personale parlamentare modellato secondo requisiti più consoni al sistema uninominale sono realmente esistenti e comuni a tutto lo spettro politico. Date per scontate le prime, ancora legate alla crisi del vecchio ceto, annovero tra le seconde la ricerca, per quello che riguarda la quota maggioritaria, di candidature spendibili sull'intera area coalizionale, ma anche il maggiore peso acquisito, rispetto al passato, dalle cariche amministrative locali (oramai tutte basate su sistemi elettorali maggioritari e rafforzate nelle competenze) rispetto alla tradizionale destinazione parlamentare della dirigenza partitica. Infine si registra un arretramento del ruolo partitico in se stesso, osservabile attraverso il ridimensionamento della quota di funzionari e di politici di professione. Usando una tipologia nota, sembra corretto indicare, a questo punto della transizione italiana, l'affermazione di alcune caratteristiche proprie del politico di carriera rispetto a quelle classiche del politico di professione (Mastropaolo 1993). L'ingresso «laterale» in politica, l'impegno non necessariamente a tempo pieno, una giovane età di elezione ed un qualificato retroterra professionale sarebbero i caratteri di questo tipo di personale, solitamente riscontrato nei parlamenti monocentrici, che utilizzerebbe dunque la parentesi politico-rappresentativa, anche lunga e impegnativa, per la propria ascesa sociale. L'ana- 
lisi precedente mostra alcuni segnali di movimento della classe politica italiana in questa direzione, dall'origine professionale più elevata e correlata con categorie sociali qualificate e «visibili», alla minore incidenza del ceto di funzionari partitico-sindacali e perfino l'abbassamento dell'età di elezione parlamentare ${ }^{24}$. Tuttavia credo che sia utile sottolineare che si tratta di un arretramento del ruolo degli apparati di partito e non di un loro superamento: in effetti, nelle due realtà partitiche chiaramente indicate dai dati come quelle più «strutturate» (rispettivamente Pds nel centro-sinistra e An nel centro-destra), la modificazione della selezione dei parlamentari non sembra rispondere ad una «cancellazione» dei vecchi percorsi interni quanto ad una graduale modifica degli stessi: nel caso del Pds si procede ad un allargamento del reclutamento in aree sociali diverse e con criteri più vasti a seconda delle circostanze e del tipo di collegio ${ }^{25}$. An sembra invece offrire un reclutamento «misto», non dissimile da quelli tradizionalmente presentati dalla Dc e dal Psi sotto il profilo del controllo partitico, in cui il requisito della socializzazione politica (in particolare attraverso l'acquisizione di cariche locali intermedie) e quello dell'esperienza amministrativa si sommano ad una certa «visibilità» sociale e locale del candidato destinato alla carriera parlamentare. Non è affatto un caso che una caratteristica innovativa come quella della forte presenza di accademici risulti, in questi ultimi due partiti, contenuta su livelli più vicini alla tradizione.

Un discorso a parte merita invece l'analisi del personale leghista. I nuovi padani approdati in parlamento mostrano e rafforzano l'idea di politici di carriera sicuramente più esperti e professionali rispetto a quelli che, oramai dieci anni or sono, costituirono la avanguardia storica di un fenomeno inaspettata-

24 Come abbiamo visto l'età media di prima elezione continua in Italia ad essere più alta di quei 40 anni che costituirebbero, secondo Mastropaolo, la soglia che distingue un politico di carriera da un politico di professione o di apparato. Tuttavia la comparazione tra gruppi specifici, per esempio quella tra Dc e Forza Italia, o quella tra Pci e Pds, mi porta a sostenere che in alcuni significativi settori della classe politica il training politico pre-parlamentare è notevolmente diminuito, ed è sostituito da un requisito di «visibilità sociale» del candidato.

25 I dati ci dicono che il Pds di oggi mostra una classe politica non troppo dissimile da quella del Pci pur rinunciando all'intera generazione dei dirigenti protagonisti delle stagioni precedenti alla svolta di fine anni ' 80 (con l'unica eccezione di Nilde Iotti). Inoltre dobbiamo ricordare che una certa parte di quella dirigenza riveste oggi ruoli centrali sia nei governi locali che nell'esecutivo nazionale (i casi di Bassolino o di Napolitano). 
mente persistente. L'idealtipo del rappresentante leghista è infatti oggi un politico presente sul territorio, cioè nella piccola amministrazione locale e nel partito cittadino. Un puro del carroccio $^{26}$, con origini socio-professionali radicate nei settori produttivi della società e selezionato per la sua capacità di operare nel collegio e nel «popolo leghista». Questo modello continua ad essere difficilmente assimilabile ai tipi tradizionali di selezione del personale, per il suo indiscutibile legame con il microcosmo sociale di riferimento, ma anche agli antipodi rispetto ai nuovi modelli che vedrebbero protagoniste strutture come il partito «del leader» o quello «mediale» ${ }^{27}$. Si tratta invece di una mediazione politica che trova riscontro solo in aree circoscritte, traendo il massimo beneficio dai meccanismi maggioritari e da una protesta anti-partitica che si associa al messaggio federalista-secessionista della attuale strategia di Bossi ${ }^{28}$.

Queste considerazioni anticipano tuttavia la risposta al secondo quesito posto poc'anzi: mi pare evidente che il processo di trasformazione dei criteri di reclutamento della classe politica sia tutt'altro che esaurito e, soprattutto, rimanga, a quattro

26 Il personale leghista è, alla luce delle informazioni sin ora raccolte, quello con la minore incidenza di esperienze partitiche precedenti: soltanto in sei casi (sul totale di 59 deputati e 29 senatori) abbiamo riscontrato una affiliazione partitica precedente da parte degli eletti del carroccio. Un dato molto diverso da quello di Forza Italia, che recluta alcuni ex parlamentari del centro-sinistra, un numero di ex politici locali e molti «semi-professionisti» di un tempo (tra cui accademici e giornalisti con noti trascorsi politici) provenienti da aree che spaziano dalla nuova sinistra al vecchio Msi.

$27 \mathrm{Si}$ discute molto su queste ed altre possibili definizioni di una forma che costituirebbe la vera alternativa ai tipi classici di organizzazioni partitiche, con particolare riferimento al caso di Forza Italia. Per quello che riguarda l'analisi del ceto politico, mi sentirei di confermare alcune peculiarità di questa formazione, che vengono anche messe in rilievo dalle analisi specifiche (Maraffi 1996). In particolare, la centralità della selezione ed il peso di un accurato lavoro di coordinamento che finisce per imporre candidature molto vicine alla leadership. Al tempo stesso segnalo che, per motivi strategici, il coordinamento funziona soltanto su un segmento ridotto della classe parlamentare. Dopo aver pagato ancora una volta il prezzo dell'alleanza, con la concessione di molti collegi «buoni» ad altre liste (in particolare Ccd e Cdu), Forza Italia concede anche circa metà dei seggi della propria quota a personaggi provenienti da movimenti ancora formalmente autonomi e comunque esclusi dal coordinamento politico del movimento (ex radicali, ex liberali e democristiani della Federazione liberal-democratica, ex leghisti).

28 Questa interpretazione trova conforto in molta della recente letteratura sul caso leghista. Ma vorrei citare in questo senso la relazione di Ilvo Diamanti al recente convegno annuale della Società Italiana di Scienza Politica (Urbino, giugno 1996), nella quale sono stati messi in rilievo in modo suggestivo i caratteri dello spiccato orientamento localistico e del forte contatto con aree delimitate, da parte del nuovo personale leghista. 
anni dall'inizio della crisi italiana, assolutamente disomogeneo. L'analisi dei caratteri socio-professionali mostra in primo luogo proprio la diversità nelle strategie di reclutamento da parte degli attori politici. Nel 1996 tale diversità sembra essersi accentuata, mostrando la profonda differenza tra i percorsi riscontrabili nella coalizione dell'Ulivo rispetto a quelli del Polo e, quindi, una sostanziale differenziazione nei caratteri della classe politica, che genera, in virtù della distribuzione geografica dei seggi, evidenti fenomeni di sovra/sotto rappresentazione di alcune categorie $^{29}$. Tuttavia, parlo di percorsi, evitando il termine modelli e riferendomi a strategie tendenziali perseguite non da attori partitici ma da vaste coalizioni ancora tutt'altro che consolidate. Ciò che sembra evidente è un obiettivo comune ai vari percorsi, e quindi un possibile elemento di unità per le future élites: l'esigenza di superare il modello tradizionale del politico professionista con un nuovo modello connotato, al tempo stesso, da caratteri di visibilità sociale e da una esperienza politicoamministrativa sufficiente per mantenere il proprio consenso. All'interno di questo quadro mi pare evidente la mancanza di tenuta di un modello di selezione sostanzialmente alternativo a quello partitico, o comunque il suo ridimensionamento all'interno di una componente politica importante ma minoritaria come quella di Forza Italia: il difficile consolidamento degli improvvisati politici del flash movement protagonista della vittoria del 1994 mostra questo limite, che dovrà essere verificato anche sugli attuali componenti del partito azienda oggi promossi in parlamento. Rimane comunque evidente che questo gruppo, pur costituendo la leadership di un attore importantissimo e una parte rilevante della nuova classe politica, non ha costituito un modello esportabile nelle altre componenti dell'alleanza di centro-destra. Stessa cosa sembra profilarsi per il seppur congruo numero di «non professionisti» schierati nel campo dell'Ulivo, qualitativamente sottomessi alle risorse dei politici puri ancora forti nel Pds, nel Ppi ed anche in alcuni cespugli della coalizione.

29 Riaggregando in tre grandi categorie i dati relativi all'origine professionale (rappresentanti del settore pubblico, di quello privato e politici-sindacalisti di professione), rileviamo un netto-squilibrio nella distribuzione territoriale: il settore privato è molto più forte al nord $(60 \%)$ e nelle regioni meridionali e insulari $(51 \%)$. Al centro invece tale dato si assesta sotto il $40 \%$, molto vicino a quello relativo al settore pubblico $(35 \%)$ e al dato relativo ai funzionari politico-sindacali $(31 \%)$. 
Il destino comune per i vari settori della classe politica italiana è allora quello di riconsolidare il rapporto tra attori organizzativi (partiti, comitati elettorali, cartelli) ed offerta di personale politico, ovvero ridefinire alcuni modelli di reclutamento politico. Il consolidamento di una nuova classe parlamentare passa necessariamente per questa strada, che implica, all'interno di ogni movimento, una certa stabilizzazione dei caratteri sociopolitici del personale. La conseguenza successiva sarà poi quella della nuova acquisizione, da parte della classe parlamentare, di un dato livello di professionismo politico. Certamente il peso delle organizzazioni partitiche e le strategie di ricerca del consenso perseguite da ogni attore influiranno in modo diverso sui possibili tipi di reclutamento: seguendo un recente contributo teorico possiamo pensare che il professionalismo della classe politica italiana risenta in particolare della presenza di managers e di staff professionals, inglobati in strutture partitiche più leggere (von Beyme 1996). Tuttavia la persistenza di forme organizzative che, a dispetto dei loro nomi stravaganti, continuano ad essere partiti nazionali centralizzati, e la scarsa territorializzazione delle campagne elettorali, fanno ritenere che in molti settori dello spettro politico assisteremo al consolidamento di un personale rappresentativo «misto». È inoltre prevedibile che alcune differenziazioni già evidenziate tra i vari gruppi possano permanere, in virtù degli effetti di una legge elettorale che, se non intervengono misure correttive sostanziose, favorisce certamente le coalizioni ma non i processi di integrazione tra i partiti. Nella quota maggioritaria potrebbero rimanere allora molti «non professionisti» di area mentre in quella proporzionale per l'elezione della camera bassa i partiti possono continuare a schierare le proprie élite centrali. Questa possibile prospettiva, se pone, da una parte, un limite ad ipotesi a mio parere esagerate circa l'evoluzione complessiva verso una classe politica più «leggera» e mutevole, sensibile alla continua riformulazione di domande da parte della società civile, nonché «territorializzata» per effetto del sistema uninominale, dall'altra porterebbe ad una conseguenza non meno importante: il differente peso specifico dell'élite parlamentare rispetto a quella partitica ${ }^{30}$ ed un re-

30 Un tipo di analisi che torna utile è quella relativa alla «sovrapposizione» degli incarichi e dei percorsi riscontrabili tra carriera partitica e parlamentare nei vari partiti. Maurizio Cotta, utilizzando questa analisi per mettere a fuoco i modelli di reclutamento della classe politica nella prima repubblica (1979), scopriva un elemento di unità (la 
lativo processo di autonomizzazione della prima dalla seconda che in qualche modo può essere letto già osservando la cronaca parlamentare degli ultimi anni ${ }^{31}$.

Ciò che invece sembra distinguere, almeno in linea tendenziale, il modello di reclutamento del personale dei due poli è l'intensità del processo di socializzazione politica e il livello di professionalizzazione. Facendo ricorso alla concettualizzazione avanzata da Cotta (1979), direi che nel personale del centro-sinistra (con particolare riferimento a quello che rappresentano Pds e Rc) permangono segnali evidenti di una alta professionalizzazione di carriera, mentre in quello proveniente dai partiti cattolici del polo e da An è la professionalizzazione di attività a prevalere. Rispetto a queste varianti che rientrano comunque in un tipo di reclutamento connotato da un elevato livello di professionismo politico, si discosta il caso di Forza Italia, nel quale abbiamo osservato le conferme del ricorso ad bomines novi (Recchi 1996) e della separazione tra dirigenza partitica e classe parlamentare. Infine dobbiamo tenere distinto un terzo possibile tipo, costruito sui caratteri indicati dal ristretto ma significativo personale parlamentare della Lega Nord. Nel complesso direi allora che le prospettive di consolidamento della classe politica per i prossimi anni ruotano oggi intorno a tre tipi teorici di professionalizzazione della classe parlamentare:

1) Professionalizzazione a controllo partitico. Si tratta del modello più continuativo rispetto al passato, poiché sia l'accesso nell'élite politica che le prospettive di carriera della stessa sono comunque legate alla selezione operata dalle dirigenze partitiche centrali. Le novità sono rappresentate soprattutto dall'accorciamento delle carriere pre-parlamentari e dalla possibilità del reclutamento per vie «laterali» che spinge in parlamento un cospicuo numero di outsiders, spesso in competizione con le stesse élite partitiche. I partiti eredi del vecchio ordine si

prevalenza della carriera partitica precedente al mandato parlamentare) con diverse varianti, legate all'intensità ed alla lunghezza della esperienza partitica pre-parlamentare. Oggi non dispongo di dati per comparare l'attuale situazione al passato, ma è evidente che la delegittimazione dei partiti e l'introduzione del collegio uninominale producono un arretramento della componente parlamentare riconducibile alla dirigenza partitica.

31 Alcuni evidenti fenomeni possono essere letti in questa prospettiva, dalla proliferazione dei gruppi parlamentari, alcuni dei quali non-partitici, alla fluidità degli stessi durante la prima legislatura maggioritaria (Verzichelli 1996), al ricorso (sempre infruttuoso) da parte di molti parlamentari incombenti a liste «fai da te», sia nel 1994 che nel 1996. Gli effetti di tali fenomeni dovranno essere valutati con più attenzione in futuro, al termine della fase di transizione. 
orienterebbero verso tale prospettiva, ma con le distinzioni importanti che menzionavo sopra, che dividono in generale le formazioni di sinistra rispetto a quelle del centro-destra, ed in particolare Rifondazione Comunista (e in misura inferiore il Pds) rispetto ad An.

2) Professionalizzazione a controllo societario. In questo caso il ruolo partitico si concentrerebbe essenzialmente nella fase di selezione del personale, producendo una classe parlamentare sempre più esperta ma autonoma rispetto alla leadership del movimento, con alcuni caratteri del politico di carriera destinato a mantenere i rapporti sociali ed anche a tornare ad essi a tempo pieno. L'estensione e il livello di centralizzazione del partitomovimento che cerca questa professionalizzazione possono determinare poi sviluppi molto diversi nel tipo di reclutamento parlamentare. L'unico partito che mostra tuttavia una propensione per questo tipo è oggi Forza Italia, una organizzazione dove gli uomini del leader «sono» il partito e quindi gli unici che possono gestire la socializzazione politica del personale parlamentare. Ad ogni modo rimane anche per queste forze il problema della visibilità sociale del rappresentante, cioè dei requisiti extra-politici che garantiscono appunto l'affidabilità della classe parlamentare per un elettorato potenzialmente molto più vasto ed eterogeneo rispetto a quello del partito in cui si identifica il candidato.

3) Professionalizzazione a controllo locale. In questo caso l'esperienza partitica riveste sicuramente un'importanza centrale nella costruzione della classe dirigente. $\mathrm{Ma}$ è la militanza, più che la carriera partitica, il requisito valido per la selezione, così come è l'esperienza diretta sul territorio, generalmente attraverso l'acquisizione di cariche esecutive locali, a produrre la visibilità necessaria per tentare l'esperienza parlamentare. Per queste caratteristiche il modello del controllo locale si addice appunto ad un personale parlamentare espressione di un attore politico isolato e poco propenso alla cooperazione, che propone una leadership ed un atteggiamento politico già sperimentato «sul campo» e, soprattutto, una rappresentanza perfettamente identificata con il collegio o con aree addirittura più ridotte, che costituiscono il «nocciolo» del consenso per il movimento che propone tale candidatura.

Stando ai dati qui presentati, il primo modello prevarrebbe tra i parlamentari del centro-sinistra (soprattutto grazie all'apporto dei partiti eredi del Pci) e il secondo (grazie a Forza Ita- 
lia) nel Polo per le libertà. Ma la chiara diversità riscontrabile anche all'interno degli schieramenti (per esempio quella tra il personale di An e di Fi) può portare, con l'ulteriore evoluzione del quadro politico, al consolidarsi dei modelli «misti» che vanno prefigurandosi. Il tempo ci dirà se tali modelli saranno realmente applicabili o se l'ulteriore aggiustamento del sistema partitico (magari sommato a nuove parziali riforme della legge elettorale) comporterà lo sviluppo di nuove soluzioni.

\section{Riferimenti bibliografici}

Bardi, L. (1996), Anti-Party Sentiment And Party System In Italy, in «European Journal of Political Research», 29, n. 3, pp. 345-363.

Beyme von, K. (1996), The Concept of Political Class: a New Dimension of Research on Elites?, in «West European Politics», XIX, n. 1, pp. 68-87.

Cotta, M. (1976), Classe politica e istituzionalizzazione del parlamento. 1946-1976, in «Rivista Italiana di Scienza Politica», VI, n. 1, pp. 71-110.

- (1979), Classe politica e parlamento in Italia, Bologna, Il Mulino.

Cotta, M. e G. Di Palma (1986) Cadres, Peones and Entrepreneurs. Professional Identities in a Divided Parliament, in E.N. Suleiman (a cura di), Parliaments and Parliamentarians in Democratic Politics, New York, Holmes \& Meier.

Cotta, M. e L. Verzichelli (1996), La classe politica: cronaca di una morte annunciata?, in M. Cotta e P. Isernia (a cura di), Il Gigante dai piedi di argilla. La crisi del regime partitocratico in Italia, Bologna, Il Mulino, pp. 373-408.

D'Alimonte, R. e A. Chiaramonte (1994), Il nuovo sistema elettorale. Quali opportunità?, in «Rivista Italiana di Scienza Politica», XXIII, n. 3, pp. 513-547.

Fabbrini, S. (1996), Luci ed ombre della transizione, in «Il Mulino», XLV, n. 2, pp. 458-467.

Gray, L. e W. Howard (1995), Forza Italia. Il partito americano, in M. Fedele e R. Leonardi (a cura di), La politica senza i partiti, Roma, Seam.

Katz, R.S. (1996), Electoral Reforms and the Transformation of Party Politics in Italy, in «Party Politics», II, n. 1, pp. 31-53.

Katz, R.S. e P. Mair (1995), Changing Models of Party Organization and Party Democracy: the Emergence of the Cartel Party, in «Party Politics», I, n. 1, pp. 5-28.

Lanza, O. (1995), Gli eletti, il ricambio deiparlamentaxinarno (1995).

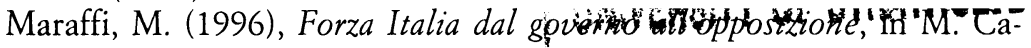


ciagli e D.I. Kertzer (a cura di), Politica in Italia. I fatti dell'anno $e$ le interpretazioni. Edizione '96, Bologna, Il Mulino.

Mastropaolo, A. (1990), Parlamenti e parlamentari negli anni ottanta, in «Rivista Italiana di Scienza Politica», XX, n. 1, pp. 29-71.

- (1993), Il ceto politico. Teoria e pratica, Roma, NIS.

- (1994), Le elezioni politiche del marzo 1994. Vecchio e nuovo nel parlamento italiano, in «Italia Contemporanea», n. 196, pp. 461470.

Mattina, L. (1994), I Candidati, in «Rivista Italiana di Scienza Politica», XXIV, n. 3, pp. 349-386.

Mény, Y. (1995), Istituzioni e Politica. Le democrazie: Germania, Stati Uniti, Francia, Gran Bretagna, Italia, Rimini, Maggioli (2a ed.).

Morlino, L. (1996), Crisis of Parties and Change of Party System in Italy, in «Party Politics», II, n. 1, pp. 5-30.

Pasquino, G. (a cura di) (1995), L'alternanza inattesa. Le elezioni del 27 marzo 1994 e le loro conseguenze, Soveria Mannelli, Rubbettino.

Pedersen, M. (1976), Political Development and Elite Transformation in Denmark, Sage Professional Paper in Contemporary Political Sociology, vol. 2.

Pizzorno, A. (1996), Definizioni del Nuovo nella Politica Italiana, in G. Galli (a cura di), La mobilità della Società Italiana. Le persone, le imprese, le istituzioni, Roma, Centro Studi Confindustria.

Recchi, E. (1996), Fishing from the Same Schools: Parliamentary Recruitment and Consociationalism in the First and Second Italian Republic, in «Western European Politics», XIX, n. 2, pp. 340-359.

Somit, A., R. Wildenmann, B. Noll e A. Römmele (1994), The Victorious Incumbent. A Threat to Democracy?, Aldershot, Dartmout.

Verzichelli, L. (1994), Gli eletti, in «Rivista Italiana di Scienza Politica», XXIV, n. 3, pp. 715-739.

- (1995), I nuovi parlamentari, in P. Ignazi e R.S. Katz (a cura di), Politica in Italia, ed. 1995, Bologna, Il Mulino.

- (1996), I gruppi parlamentari dopo il 1994. Fluidità e riaggregazio$n i$, in «Rivista Italiana di Scienza Politica», XXVI, n. 2, pp. 391413.

Zincone, G. (1995), U.S.A. con cautela, Roma, Donzelli. 